BULLETIN (New Series) OF THE

AMERICAN MATHEMATICAL SOCIETY

Volume 42, Number 2, Pages 137-162

S 0273-0979(05)01047-5

Article electronically published on January 25, 2005

\title{
CONTINUED FRACTIONS AND MODULAR FUNCTIONS
}

\author{
W. DUKE
}

The mathematical universe is inhabited not only by important species but also by interesting individuals.-C.L. Siege 1

\section{INTRODUCTION}

It is widely recognized that the work of Ramanujan deeply influenced the direction of modern number theory. This influence resonates clearly in the "Ramanujan conjectures". Here I will explore another part of his work whose position within number theory seems to be less well understood, even though it is more elementary, namely that related to continued fractions. I will concentrate on the special values of continued fractions that represent modular functions, especially the Rogers-Ramanujan continued fraction. These give analogues of the simple continued fraction expansions of units in real quadratic fields. My primary motivation is to furnish a coherent treatment of this topic, around which an air of mystery seems to linger. Another is to provide an inviting and non-standard introduction to the classical theory of modular functions.

This is largely an expository paper; most of the ideas I discuss are well known. Yet it is hoped that the elaboration given here combines these ideas in a novel way. Although this paper is not intended to be comprehensive, its later sections contain more material than is likely needed to gain a clear impression of the main themes, which the first six sections should provide. These will take the general reader through a proof of the first main result, Theorem 1, introducing the needed concepts along the way. The sections that follow these assume a somewhat greater background in number theory.

\section{The Rogers-Ramanujan CONTINUEd FRACtion}

According to G.H. Hardy [Har, p. 13], Ramanujan's masterpiece in continued fractions was his work on the Rogers-Ramanujan continued fraction. This is defined by

$$
r(\tau)=\frac{q^{1 / 5}}{1+\frac{q}{1+\frac{q^{2}}{1+\frac{q^{3}}{1+\cdots}}}}=\frac{q^{1 / 5}}{1+} \frac{q}{1+} \frac{q^{2}}{1+} \frac{q^{3}}{1+\ldots},
$$

Received by the editors November 4, 2003.

2000 Mathematics Subject Classification. Primary 11Fxx, 11Gxx.

Research supported in part by NSF Grant DMS-0355564.

${ }^{1}$ See Mag, p. 10]. 
where $q=e(\tau)=e^{2 \pi i \tau}$. It converge ${ }^{2}$ for $\tau \in \mathcal{H}$, the upper half-plane, and also for some $\tau \in \mathbb{R}$, for example $\tau=0$, where

$$
r(0)=\frac{1}{1+} \frac{1}{1+} \frac{1}{1+} \frac{1}{1+\ldots}=\frac{-1+\sqrt{5}}{2} .
$$

In his first letter to Hardy in 1913 [Ram1, p. xxvii], Ramanujan gave a remarkable analogue of (2.2) for the value at $i=\sqrt{-1}$ :

$$
r(i)=\frac{e^{\frac{-2 \pi}{5}}}{1+} \frac{e^{-2 \pi}}{1+} \frac{e^{-4 \pi}}{1+} \frac{e^{-6 \pi}}{1+} \ldots=\sqrt{\frac{5+\sqrt{5}}{2}}-\frac{1+\sqrt{5}}{2} .
$$

Another value he gave in his "lost" notebook was at the cube root of unity $\rho=$ $\frac{-1+\sqrt{-3}}{2}$, which can be written

$$
e\left(\frac{1}{10}\right) r(\rho)=\frac{e^{\frac{-\pi \sqrt{3}}{5}}}{1-} \frac{e^{-\pi \sqrt{3}}}{1+} \frac{e^{-2 \pi \sqrt{3}}}{1-} \frac{e^{-3 \pi \sqrt{3}}}{1+} \ldots=\frac{\sqrt{30+6 \sqrt{5}}-3-\sqrt{5}}{4} .
$$

In his letter he stated that $r\left(\frac{1}{2} \sqrt{-n}\right)$ "can be exactly found if $n$ be any positive rational quantity" and in his notebooks and other writings provided several more examples. Ramanujan's writings do not contain proofs of any of these results ${ }^{3}$ In his lecture on Ramanujan in 1988 [Se2 p. 699], Selberg wrote

One might speculate, although it may be somewhat futile, about what would have happened if Ramanujan had come in contact not with Hardy but with a great mathematician of more similar talents, someone who was more inclined in the algebraic directions, for instance E. Hecke in Germany.

One goal of this paper is to show that special evaluations of the Rogers-Ramanujan continued fraction are best understood through Klein's theory of the icosahedron and its connection with modular functions. Klein's theory, already articulated in 1877-1878 in [K11] and [K12], together with basic facts from the classical theory of complex multiplication, neatly explain these results.

We will see in 9 below that the Rogers-Ramanujan continued fraction $r=r(\tau)$ satisfies the icosahedral equation

$$
\left(r^{20}-228 r^{15}+494 r^{10}+228 r^{5}+1\right)^{3}+j(\tau) r^{5}\left(r^{10}+11 r^{5}-1\right)^{5}=0,
$$

where $j$ is the classical modular function

$$
j(\tau)=q^{-1}+744+196884 q+21493760 q^{2}+\cdots .
$$

This fact has as a consequence the following result, whose proof is completed at the end of 4 ;

Theorem 1. The value $r(\tau)$ can be expressed in terms of radicals over $\mathbb{Q}$ if and only if $j(\tau)$ can and the icosahedral equation (2.5) is reducible over $\mathbb{Q}(\zeta, j(\tau))$, where $\zeta=e\left(\frac{1}{5}\right)$.

\footnotetext{
${ }^{2}$ For background on continued fractions see $[\mathrm{BC}$, Chap. XV]. A more advanced reference is Per.

${ }^{3}$ Proofs of 2.3) and (2.4) were first published in Wa1 and [Ra2], respectively. Watson's reconstruction of Ramanujan's line of thought leading to (2.3) is nicely described in [MM, p. 154]. Proofs of several of Ramanujan's other claims about special values of $r(\tau)$ may be found in Ra1] and $[\mathrm{BCZ}]$.
} 
Recall that an algebraic number is a root of an integral polynomial, an algebraic integer is a root of a monic integral polynomial and that a unit is an algebraic integer whose inverse is also an algebraic integer. Let $\overline{\mathbb{Q}}$ denote the field of all algebraic numbers. A number field is a subfield of $\overline{\mathbb{Q}}$ of finite degree over $\mathbb{Q}$. An imaginary quadratic field is a number field of degree 2 over $\mathbb{Q}$ that is not contained in $\mathbb{R}$.

It follows easily from (2.5) that $r(\tau)$ is a unit if $j(\tau)$ is an algebraic integer. In his classic book of 1908 Weber Web p. 423] proved the fundamental result in the theory of complex multiplication that $j(\tau)$ is an algebraic integer if $\tau$ is in an imaginary quadratic field. Thus in this case $r(\tau)$ is a unit 4 Furthermore, $j(\tau)$ can be expressed in terms of radicals over $\mathbb{Q}$. We recall these facts and complete the proof of the following result in 8 .

Theorem 2. If $\tau$ is in an imaginary quadratic field, then $r(\tau)$ is a unit that can be expressed in terms of radicals over $\mathbb{Q}$.

Perhaps this provides a satisfactory interpretation of Ramanujan's general claim about $r\left(\frac{1}{2} \sqrt{-n}\right)$. For $\tau$ imaginary quadratic, 2.5) reduces the evaluation of $r(\tau)$ to a machine calculation. Thus $r(i)$ from (2.3) is a root of the factor $r^{4}+2 r^{3}-6 r^{2}-2 r+1$ of (2.5) when $j(i)=1728$. As another example we have

$$
\begin{aligned}
\frac{e^{\frac{-\pi \sqrt{19}}{5}}}{1-} \frac{e^{-\pi \sqrt{19}}}{1+} \frac{e^{-2 \pi \sqrt{19}}}{1-} & \frac{e^{-3 \pi \sqrt{19}}}{1+} \ldots \\
& =\frac{-8-3 \sqrt{5}-\sqrt{125+60 \sqrt{5}}+\sqrt{250+108 \sqrt{5}+(16+6 \sqrt{5}) \sqrt{125+60 \sqrt{5}}}}{4},
\end{aligned}
$$

which comes from solving (2.5) when $j\left(\frac{-1+\sqrt{19}}{2}\right)=-2{ }^{15} 3^{3}$. The only limitations to evaluating $r(\tau)$ for $\tau$ imaginary quadratic are one's software and patience, since $j(\tau)$ is explicitly computable. On the other hand, if $\tau$ has $j(\tau)=1$, say, then $r(\tau)$ is a unit but it is not expressible in terms of radicals over $\mathbb{Q}$.

Some unexpected consequences of the connection of $r(\tau)$ with the icosahedron, proven at the end of \$3], are the identity

$$
r\left(\frac{-7 \tau-10}{5 \tau+7}\right)=\frac{-1}{r(\tau)},
$$

and the simple evaluations

$$
r\left(\frac{7+i}{10}\right)=i \text { and } r\left(\frac{-7+i}{5}\right)=-i .
$$

There is also a sort of converse result, proven in 48 , that states that the evaluations given in (2.7) are the simplest possible for an algebraic argument.

Theorem 3. The only values of $r(\tau)$ that are algebraic of degree $\leq 2$ over $\mathbb{Q}$ for an algebraic value of $\tau \in \mathcal{H}$ are $\pm i$, as in (2.7).

The following six sections contain proofs of these results as well as a number of other identities involving the Rogers-Ramanujan continued fraction. In $\$ 9$ we provide a parallel (but abbreviated) treatment of other continued fractions and then in $\$ 10$ give a brief discussion of the role of a certain generalization of continued fractions in the theory of modular functions.

\footnotetext{
${ }^{4}$ This fact (for $\tau=\frac{1}{2} \sqrt{-n}$ ) was first proven in [BCZ] in another way.
} 


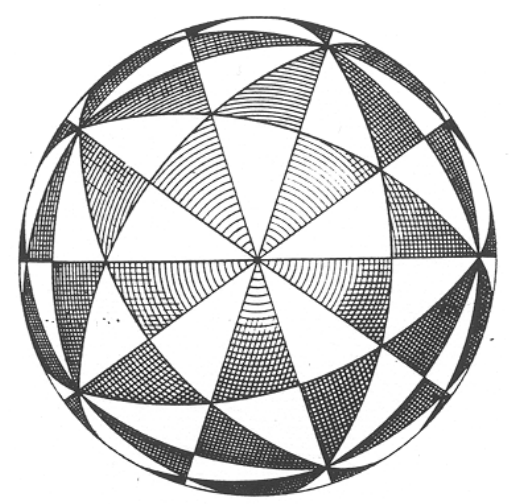

Figure 1. Icosahedral tessellation of the sphere.

\section{THE ICOSAHEDRON}

There is a beautiful connection between the Rogers-Ramanujan continued fraction (2.1) and the icosahedron that explains the evaluations (2.3) and (2.4) geometrically but that, surprisingly, does not appear to be widely known. It also provides the first step toward proving (2.5) and Theorem 1 .

Consider an icosahedron inscribed in the unit sphere and subdivide each face into 6 triangles by connecting its centroid with the surrounding vertices and edge midpoints. First project these points and edges onto the sphere from its center, resulting in a tessellation of the sphere into triangles and place the sphere over the complex plane as in Figure 15 shown from directly above.

Next, stereographically project this tessellation on the extended complex plane and call a resulting point of intersection an edge point, a face point or a vertex depending on its origin in the icosahedron. As shown in Figure 2 an edge point, face point or vertex has 4,6 or 10 triangles coming into it, respectively.

The following result shows that the Rogers-Ramanujan continued fraction plays a role for the icosahedron that is analogous to that played by the exponential function for a regular polygon.

Proposition 1. As a $, b, c, d$ run over all integers with $a d-b c=1, r\left(\frac{a i+b}{c i+d}\right)$ runs over the edge points and $r\left(\frac{a \rho+b}{c \rho+d}\right)$ runs over the face points. Furthermore, $r\left(\frac{a \cdot 0+b}{c \cdot 0+d}\right)=r\left(\frac{b}{d}\right)$ converges if and only if $5 \nmid d$, in which case $r\left(\frac{b}{d}\right)$ runs over the non-zero finite vertices.

The proof of this relies on the group of proper rotations of the sphere that leave the icosahedron invariant. This group is well-known to be isomorphic to $A_{5}$, the alternating group of order 60 . Each of the 59 non-trivial rotations is of order 2, 3 or 5 and has two fixed points, whose images in the extended complex plane are both edge points, face points, or vertices, respectively. This group corresponds under

\footnotetext{
${ }^{5}$ The figures in this paper are all reproduced from Fricke [Fri].
} 


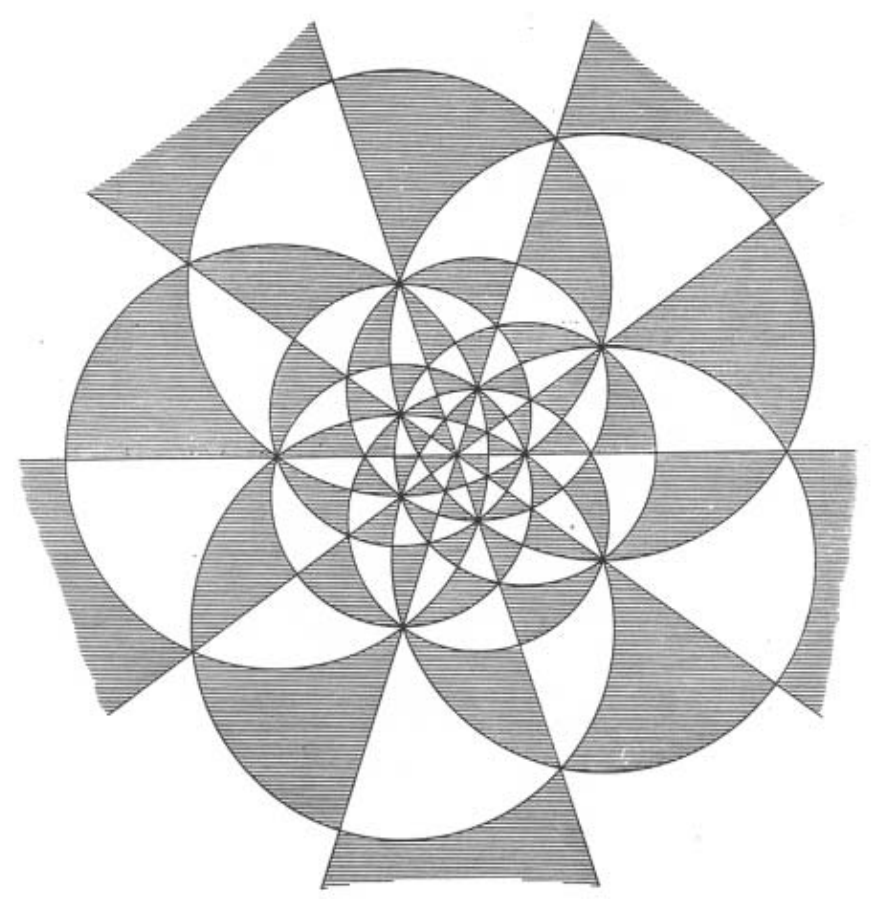

FiguRE 2. Stereographic projection of icosahedral tessellation.

stereographic projection to the subgroup $G_{60} \subset \operatorname{PSL}(2, \mathbb{C})$ of Möbius transformations with generators represented in $S U(2)$ by the matrices

$$
S=\left(\begin{array}{cc}
\zeta^{3} & 0 \\
0 & \zeta^{2}
\end{array}\right) \text { and } T=\frac{1}{\sqrt{5}}\left(\begin{array}{cc}
\zeta-\zeta^{4} & \zeta^{3}-\zeta^{2} \\
\zeta^{3}-\zeta^{2} & \zeta^{4}-\zeta
\end{array}\right)
$$

where $\zeta=e\left(\frac{1}{5}\right)$, giving elliptic transformations of order 5 and 2 , respectively Fri. p. 42] (or [Tot, p. 28]). The group $G_{60}$ also contains the elliptic transformation of order 3 represented by

$$
W=T S=\frac{1}{\sqrt{5}}\left(\begin{array}{cc}
\zeta^{4}-\zeta^{2} & 1-\zeta^{4} \\
\zeta-1 & \zeta-\zeta^{3}
\end{array}\right)
$$

Clearly the vertices 0 and $\infty$ are the fixed points of $S$. Using the elementary relations

$$
\zeta+\zeta^{4}=-\left(\zeta^{2}+\zeta^{3}\right)^{-1}=\frac{-1+\sqrt{5}}{2} \text { and } \frac{\zeta-\zeta^{4}}{\zeta^{2}-\zeta^{3}}=\frac{1+\sqrt{5}}{2},
$$

it is straightforward to compute the fixed points of $T$ and $W$ : they are

$$
t_{ \pm}=-\frac{1+\sqrt{5}}{2} \pm \sqrt{\frac{5+\sqrt{5}}{2}} \text { and } w_{ \pm}=\zeta^{2} \frac{3+\sqrt{5} \pm \sqrt{30+6 \sqrt{5}}}{4} .
$$

We may identify the (non-Euclidean) triangle formed by $0, t_{+}$and $w_{-}$as the small shaded triangle of Figure 2 with 0 as a vertex and an edge along the positive real axis. Its internal angles are $\frac{\pi}{2}, \frac{\pi}{3}$ and $\frac{\pi}{5}$. Another way of arriving at a transformation of $G_{60}$ is to perform an even number of reflections in the sides of this triangle and its images.

To prove Proposition 1 we need the connection between $G_{60}$ and the modular group $\Gamma(1)=\operatorname{PSL}(2, \mathbb{Z})$. As usual, $g \in \Gamma(1)$ acts on $\tau \in \mathcal{H}$ as a linear fractional 
transformation. For any positive integer $N$ let $\Gamma(N) \subset \Gamma(1)$ be the normal subgroup consisting of those transformations in $\Gamma(1)$ that are congruent to the identity mod $N$. This subgroup is known as the principal congruence subgroup of level $N$.

Proposition 2. Suppose $\tau \in \mathcal{H}$. We have that

$$
\begin{aligned}
& r(\tau+1)=\operatorname{Sr}(\tau)=\zeta r(\tau) \quad \text { and } \\
& r\left(\frac{-1}{\tau}\right)=\operatorname{Tr}(\tau)=\frac{-(1+\sqrt{5}) r(\tau)+2}{2 r(\tau)+1+\sqrt{5}} .
\end{aligned}
$$

Also, $r(g \tau)=r(\tau)$ for all $g \in \Gamma(5)$.

The evaluations (2.3) and (2.4) are immediate consequences of Proposition 2 2 and (3.1), since $r(i)$ is a fixed point of $T$ and $r(\rho)$ one of $W=T S$ and the appropriate values from (3.1) are easily determined. The first statement of Proposition 1 also now follows from Proposition 2, since $r(i)$ is an edge point and $r(\rho)$ is a face point, and $G_{60}$ acts transitively on each type. Of course, any of these special values can be explicitly computed using Proposition 2 .

In 1917 Schur $[\mathrm{Sch}$ proved that $r(b / d)$ converges if and only if $5 \nmid d$. Since $r(0)$ as given in (2.2) is a finite vertex, the second statement of Proposition 1 follows as well by a simple continuity argument, thereby reducing the proof of Proposition 1 to that of Proposition 2.

In fact, when $5 \nmid d$, Schur evaluated $r(b / d)$, and his result can be put in the elegant form

$$
r\left(\frac{b}{d}\right)=\left(\frac{5}{d}\right)\left(r(0) e\left(\frac{b d}{5}\right)\right)^{\left(\frac{5}{d}\right)} .
$$

This formula for the non-zero finite vertices is also easy to derive using Proposition 2 .

The "unexpected" consequences (2.6) and (2.7) of Proposition 2 come from the fact that $G_{60}$ contains the inversion $U: z \mapsto-1 / z$, where $U=T S^{2} T S^{3} T S^{2}$ [Fri] p. 42].

\section{An identity of Rogers And theta COnstants}

Probably the most important fact about the Rogers-Ramanujan continued fraction, and the key to the proof of Proposition 2 is the famous identity proven first by Rogers Ro1]:

$$
r(\tau)=q^{1 / 5} \prod_{n \geq 1}\left(1-q^{n}\right)^{\left(\frac{5}{n}\right)}
$$

for $\tau \in \mathcal{H}$, where $\left(\frac{5}{n}\right)$ is the Kronecker symbol. This follows from the RogersRamanujan identities

$$
\begin{aligned}
& \sum_{n \geq 0} \frac{q^{n^{2}}}{(1-q) \cdots\left(1-q^{n}\right)}=\prod_{n \geq 1} \frac{1}{\left(1-q^{5 n-1}\right)\left(1-q^{5 n-4}\right)} \\
& \sum_{n \geq 0} \frac{q^{n(n+1)}}{(1-q) \cdots\left(1-q^{n}\right)}=\prod_{n \geq 1} \frac{1}{\left(1-q^{5 n-2}\right)\left(1-q^{5 n-3}\right)} .
\end{aligned}
$$

To see this, observe that the function

$$
R(z)=\sum_{n \geq 0} z^{n} q^{n^{2}}\left[(1-q) \cdots\left(1-q^{n}\right)\right]^{-1}
$$


satisfies the recurrence $R(z)=R(z q)+z q R\left(z q^{2}\right)$, so that $r(\tau)=$ $q^{1 / 5} R(q) / R(1)$. The Rogers-Ramanujan identities have purely combinatorial interpretations. For example, (4.2) says that the number of partitions of $n$ with minimal difference 2 is equal to the number of partitions into parts of the forms $5 m+1$ and $5 m+4$. See [Ask] for an account of the (complex) history of the Rogers-Ramanujan identities and references to the different proofs.

Actually, a formula equivalent to (3.2) was stated by Ramanujan in his second letter to Hardy [Ram1, p. xxviii] and a proof was first published by Watson Wa2. We will give a proof of Proposition 2 based on theta constants that better illuminates what is going on and is more general.

The theta constant with characteristic $\left[\begin{array}{c}\epsilon \\ \epsilon^{\prime}\end{array}\right] \in \mathbb{R}^{2}$ is defined by

$$
\theta\left[\begin{array}{c}
\epsilon \\
\epsilon^{\prime}
\end{array}\right](\tau)=\sum_{n \in \mathbb{Z}} e\left(\frac{1}{2}\left(n+\frac{\epsilon}{2}\right)^{2} \tau+\frac{\epsilon^{\prime}}{2}\left(n+\frac{\epsilon}{2}\right)\right)
$$

for $\tau \in \mathcal{H}$. It satisfies the following basic properties for $\ell, m, N \in \mathbb{Z}$ with $N$ positive [FK, pp. 72-77]:

$$
\begin{aligned}
& \theta\left[\begin{array}{c}
\epsilon \\
\epsilon^{\prime}
\end{array}\right](\tau)=e\left(\mp \frac{\epsilon m}{2}\right) \theta\left[\begin{array}{c} 
\pm \epsilon+2 \ell \\
\pm \epsilon^{\prime}+2 m
\end{array}\right](\tau) \\
& \left.\theta\left[\begin{array}{c}
\epsilon \\
\epsilon^{\prime}
\end{array}\right](\tau)=\sum_{k=0}^{N-1} \theta\left[\frac{\epsilon+2 k}{N}\right] \frac{N}{N \epsilon^{\prime}}\right]\left(N^{2} \tau\right) .
\end{aligned}
$$

It also satisfies for $\left(\begin{array}{l}a b \\ c d\end{array}\right) \in \mathrm{SL}(2, \mathbb{Z})$ the fundamental transformation law $\mathrm{FK}$. Thm 1.11, p. 81]:

$$
\theta\left[\begin{array}{c}
\epsilon \\
\epsilon^{\prime}
\end{array}\right]\left(\frac{a \tau+b}{c \tau+d}\right)=\kappa \sqrt{c \tau+d} \theta\left[\begin{array}{c}
a \epsilon+c \epsilon^{\prime}-a c \\
b \epsilon+d \epsilon^{\prime}+b d
\end{array}\right](\tau)
$$

where

$$
\kappa=e\left(-\frac{1}{4}\left(a \epsilon+c \epsilon^{\prime}\right) b d-\frac{1}{8}\left(a b \epsilon^{2}+c d \epsilon^{2}+2 b c \epsilon \epsilon^{\prime}\right)\right) \kappa_{0},
$$

with $\kappa_{0}$ an eighth root of unity depending only on the matrix $\left(\begin{array}{c}a b \\ c d\end{array}\right)$. The value of $\kappa_{0}$ is fixed by choosing the argument of the square root to be in $[0, \pi)$. In particular we have [FK, p. 86]

$$
\theta\left[\begin{array}{c}
\epsilon \\
\epsilon^{\prime}
\end{array}\right](\tau+1)=e\left(-\frac{\epsilon}{4}\left(1+\frac{\epsilon}{2}\right)\right) \theta\left[\begin{array}{c}
\epsilon \\
\epsilon+\epsilon^{\prime}+1
\end{array}\right](\tau)
$$

and

$$
\theta\left[\begin{array}{c}
\epsilon \\
\epsilon^{\prime}
\end{array}\right]\left(\frac{-1}{\tau}\right)=e\left(-\frac{1}{8}\right) \sqrt{\tau} e\left(\frac{\epsilon \epsilon^{\prime}}{4}\right) \theta\left[\begin{array}{c}
\epsilon^{\prime} \\
-\epsilon
\end{array}\right](\tau) .
$$

Additional transformation formulas invoked later in the text are given in Appendix A. We also have the product formula [FK, p. 141]:

$$
\theta\left[\begin{array}{c}
\epsilon \\
\epsilon^{\prime}
\end{array}\right](\tau)=e\left(\frac{\epsilon \epsilon^{\prime}}{4}\right) q^{\frac{\epsilon^{2}}{8}} \prod_{n \geq 1}\left(1-q^{n}\right)\left(1+e\left(\frac{\epsilon^{\prime}}{2}\right) q^{n-\frac{1+\epsilon}{2}}\right)\left(1+e\left(\frac{-\epsilon^{\prime}}{2}\right) q^{n-\frac{1-\epsilon}{2}}\right),
$$

which follows from the Jacobi triple product identity.

A short calculation using (4.1) and (4.8) shows that

$$
r(\tau)=e\left(-\frac{1}{10}\right) \frac{\theta\left[\begin{array}{c}
3 / 5 \\
1
\end{array}\right](5 \tau)}{\theta\left[\begin{array}{c}
1 / 5 \\
1
\end{array}\right](5 \tau)} .
$$


Since clearly $r(\tau+1)=\zeta r(\tau)$, consider (3.2). For this we have using (4.7), (4.3) and (4.4)

$$
r\left(\frac{-1}{\tau}\right)=e\left(-\frac{1}{10}\right) \frac{\theta\left[\begin{array}{c}
3 / 5 \\
1
\end{array}\right]\left(\frac{-5}{\tau}\right)}{\theta\left[\begin{array}{c}
1 / 5 \\
1
\end{array}\right]\left(\frac{-5}{\tau}\right)}=\frac{\theta\left[\begin{array}{c}
1 \\
3 / 5
\end{array}\right]\left(\frac{\tau}{5}\right)}{\theta\left[\begin{array}{c}
1 \\
1 / 5
\end{array}\right]\left(\frac{\tau}{5}\right)}=\frac{\sum_{k=0}^{4} \theta\left[\begin{array}{c}
\frac{1+2 k}{5} \\
3
\end{array}\right](5 \tau)}{\sum_{k=0}^{4} \theta\left[\begin{array}{c}
\frac{1+2 k}{5} \\
1
\end{array}\right](5 \tau)} .
$$

Using again (4.3) and its consequence that $\theta\left[\begin{array}{c}1 \\ 1\end{array}\right](\tau)=0$, we get after some simplification that

$$
r\left(\frac{-1}{\tau}\right)=\frac{\left(e\left(\frac{1}{5}\right)-e\left(\frac{4}{5}\right)\right) r(\tau)+\left(e\left(\frac{3}{5}\right)-e\left(\frac{2}{5}\right)\right)}{\left(e\left(\frac{3}{5}\right)-e\left(\frac{2}{5}\right)\right) r(\tau)-\left(e\left(\frac{1}{5}\right)-e\left(\frac{4}{5}\right)\right)}=\operatorname{Tr} r(\tau),
$$

and this can be written $r\left(\frac{-1}{\tau}\right)=\frac{-(1+\sqrt{5}) r(\tau)+2}{2 r(\tau)+1+\sqrt{5}}$.

It is straightforward to check that $r$ is invariant under $\Gamma(5)$ using either Lemma A.1 or the fact that $\Gamma(5)$ is the unique normal subgroup of index 60 in $\Gamma(1)[\mathrm{New}]$, thus finishing the proof of Proposition 2

\section{INVARIANTS AND THE ICOSAHEDRAL EQUATION}

As preparation for the proof of (2.5) and Theorem 10 we now review Klein's construction of invariants of the icosahedral group $G_{60}$. Given a finite subgroup $G \subset \operatorname{PSL}(2, \mathbb{C})$, a (projective) invariant of weight $k$ for $G$ is a rational function $I(z) \in \mathbb{C}(z)$ that satisfies

$$
I\left(\frac{a z+b}{c z+d}\right)=(c z+d)^{k} I(z)
$$

for every $\left(\begin{array}{ll}a & b \\ c & b\end{array}\right) \in G$. By using the formula for the vertices given in (3.3), it is easy to check that

$$
\prod_{b(\bmod 10)}\left(z-r\left(\frac{b}{2}\right)\right)=z^{10}+11 z^{5}-1 .
$$

Thus the polynomial $V(z)=z\left(z^{10}+11 z^{5}-1\right)$ has a simple zero at each finite vertex. It follows that $V$ is an invariant of weight -12 for $G_{60}$. The Hessian determinant of the corresponding homogeneneous form

$$
\tilde{V}(z, w)=w^{12} V(z / w),
$$

namely

$$
\tilde{F}(z, w)=\left|\begin{array}{cc}
\tilde{V}_{z z} & \tilde{V}_{z w} \\
\tilde{V}_{w z} & \tilde{V}_{w w}
\end{array}\right|,
$$

gives another invariant [Dic, p. 4]

$$
F(z)=-\frac{1}{121} \tilde{F}(z, 1)=z^{20}-228 z^{15}+494 z^{10}+228 z^{5}+1,
$$

this time of weight -20 . Since the 20 roots of this polynomial are distinct and left stable under $G_{60}$, it follows that they must be the face points. One can also check directly that $F\left(w_{+}\right)=0$, where $w_{+}$is the face point from (3.1). Finally, the Jacobian determinant

$$
\tilde{E}(z, w)=\left|\begin{array}{ll}
\tilde{V}_{z} & \tilde{V}_{w} \\
\tilde{F}_{z} & \tilde{F}_{w}
\end{array}\right|
$$

gives an invariant

$$
E(z)=\frac{1}{20} \tilde{E}(z, 1)=z^{30}+522\left(z^{25}-z^{5}\right)-10005\left(z^{20}+z^{10}\right)+1
$$


of weight -30 whose roots are the edge points. These three invariants satisfy an algebraic relation (a syzygy):

$$
E^{2}-F^{3}=1728 V^{5}
$$

For a positive integer $N$ the $N$-th cyclotomic field $\mathbb{Q}\left(\zeta_{N}\right)$ is the smallest number field containing the $N$-th root of unity $\zeta_{N}=e(1 / N)$. Now the invariant $V(z)=$ $z\left(z^{10}+11 z^{5}-1\right)$ factors over $\mathbb{Q}$ as

$$
V(z)=z\left(z^{2}+z-1\right)\left(z^{4}-3 z^{3}+4 z^{2}-2 z+1\right)\left(z^{4}+2 z^{3}+4 z^{2}+3 z+1\right) .
$$

Its splitting field, which is the smallest subfield of $\mathbb{C}$ containing all of its roots, is easily checked to be $\mathbb{Q}\left(\zeta_{10}\right)=\mathbb{Q}(\zeta)$. Furthermore, every root is a unit since the constant term of $F$ is 1 . Similarly, the splitting field of $F$ is $\mathbb{Q}\left(\zeta_{20}\right)$ while that of $E$ is $\mathbb{Q}\left(\zeta_{30}\right)$, and all of the roots are units. As a consequence of Proposition 1 we have

Corollary 1. Each of the 60 non-zero finite vertices, edge points, and face points of the icosahedron is a unit in the cyclotomic field $\mathbb{Q}\left(\zeta_{60}\right)$. Each of these units has a continued fraction expansion obtained by evaluating $r(\tau)$ for some $\tau \in \mathbb{Q}\left(\zeta_{60}\right)$. 0 :

By using the basic invariants $V$ and $F$ we may construct an invariant of weight

$$
J(z)=-\frac{F(z)^{3}}{V(z)^{5}}=-\frac{\left(z^{20}-228 z^{15}+494 z^{10}+228 z^{5}+1\right)^{3}}{z^{5}\left(z^{10}+11 z^{5}-1\right)^{5}} .
$$

The associated icosahedral equation 6

$$
\left(z^{20}-228 z^{15}+494 z^{10}+228 z^{5}+1\right)^{3}+J z^{5}\left(z^{10}+11 z^{5}-1\right)^{5}=0
$$

defines a function field extension $\mathbb{K}(z)$ over $\mathbb{K}(J)$ of degree 60 for any field $\mathbb{K} \subset \mathbb{C}$, since as a polynomial in $z$ and $J$ the left hand side of (5.4) is easily checked to be irreducible over $\mathbb{C}$. Provided $\zeta=e(1 / 5) \in \mathbb{K}$, a transformation $A$ of $G_{60}$ acts as an automorphism of the field $\mathbb{K}(z)$ by $f(z) \mapsto f\left(A^{-1} z\right)$. Let $\mathbb{L} \subset \mathbb{K}(z)$ be the fixed field of $G_{60}$. By a well-known theorem (see e.g. [Art]), $\mathbb{K}(z)$ is a Galois extension of degree 60 over $\mathbb{L}$ with Galois group $G_{60}$. Since $J \in \mathbb{L}$, we must have $\mathbb{L}=\mathbb{K}(J)$. Thus we have

Proposition 3. Suppose that $\mathbb{K} \subset \mathbb{C}$ is a field that contains $\zeta$. The field $\mathbb{K}(z)$ is a Galois extension of $\mathbb{K}(J(z))$ with Galois group $G_{60} \simeq A_{5}$.

One computes that the discriminant of the left hand side of (5.4) is

$$
5^{785} J^{40}(J-1728)^{30}
$$

and hence that the only values of $J$ for which (5.4) has multiple roots are $J=0$ and $J=1728$. By a standard result of Galois theory (see e.g. [Bra, p. 214]) we know that for any specialization of $J \in \mathbb{C}$ for which (5.4) has no multiple roots, the Galois group of $\mathbb{K}(z) / \mathbb{K}(J)$ is a subgroup of $G_{60}$, which is proper if and only if (5.4) is reducible over $\mathbb{K}(J)$. We have shown the following:

Proposition 4. Suppose that $\mathbb{K} \subset \mathbb{C}$ is a field that contains $\zeta$, that $J \in \mathbb{C} \backslash\{0,1728\}$ and that $z$ is a root of (5.4). Then $\mathbb{K}(z)$ defines a Galois extension of $\mathbb{K}(J)$ whose Galois group $G$ is a subgroup of $A_{5}$. This subgroup is proper if and only if (5.4) is reducible over $\mathbb{K}(J)$.

\footnotetext{
${ }^{6}$ This name is sometimes mistakenly applied to the syzygy (5.2).
} 
Note that by (5.2) we have an equivalent alternative to (5.4):

$$
E^{3}(z)+(J(z)-1728) V(z)^{5}=0
$$

Thus the cyclotomic fields generated by face points and edge points treated in Proposition 1 correspond to the only ramified specializations $J=0$ and $J=1728$, respectively.

A special case of a major result of Klein [K14] (see also [Tot p. 80]) implies that the splitting field of any principal quintic

$$
x^{5}+a_{2} x^{2}+a_{1} x+a_{0}
$$

defined over $\mathbb{K}(J)$ with Galois group $A_{5}$ is of the form $\mathbb{K}(z)$ for some $J \in \mathbb{K}$. This result of Klein is the point of departure for Serre's work on the Witt invariant of the trace form $\operatorname{Tr}\left(x^{2}\right)$ [Ser1, Ser2, Ser3].

\section{Modular Functions}

Turning now to the proof of (2.5) and Theorem 1 it is finally time to introduce modular functions. A congruence subgroup $\Gamma$ of $\Gamma(1)$ of level $N$ is a subgroup that contains $\Gamma(N)$ for some $N$. Given a congruence subgroup $\Gamma$ the quotient $\Gamma \backslash \mathcal{H}$ can be made into a Riemann surface with a finite number of punctures occurring at the inequivalent cusps, which are the fixed points of the parabolic transformations of $\Gamma$, hence rational numbers (and $i \infty$ ). By filling in these punctures we obtain a compact Riemann surface whose genus can be computed from $\Gamma$ [Sc, p. 93].

A modular function $f$ for $\Gamma$ is a meromorphic function on this compact Riemann surface. This means that $f$ is meromorphic on $\mathcal{H}, f(g \tau)=f(\tau)$ for all $g \in \Gamma$ and that for every $g \in \Gamma(1)$ the Laurent expansion

$$
f(g \tau)=\sum_{n \in \mathbb{Z}} a_{g}(n) q^{n / w_{g}}
$$

around the cusp $g^{-1}(i \infty)$ has only finitely many nonzero negative coefficients. Here $w_{g} \mid N$ is the width of the cusp, which is the smallest $m \in \mathbb{Z}^{+}$so that

$$
\pm g^{-1}\left(\begin{array}{ll}
1 & 1 \\
0 & 1
\end{array}\right)^{m} g \in \Gamma \text {. }
$$

The order of a pole or zero at the cusp is measured in terms of $q^{1 / w_{g}}$. Since $i \infty$ is always a cusp there is a distinguished Laurent expansion, often called simply the $q$-expansion of $f$ :

$$
f(\tau)=\sum_{n} a_{1}(n) q^{n / w_{1}}=\sum_{n} a(n) q^{n / w} .
$$

The $a(n)$ so defined are called the Fourier coefficients of $f$.

Figure 3 shows the familiar tessellation of $\mathcal{H}$ by the action of the full modular group $\Gamma(1)$, a fundamental domain for this action being any pair of triangles, one shaded and one not. Now $\Gamma(1)$ has only 1 cusp, the cusp at $i \infty$, which has width 1 , and its associated compact Riemann surface has genus 0 . Thus there is a unique modular function for $\Gamma(1)$ that is holomorphic on $\mathcal{H}$ with a simple pole at $i \infty$, that vanishes at $\tau=\rho$, and that takes the value $1728=12^{3}$ at $\tau=i$. This modular function may be explicitly given by either of the following formulas:

$$
j(\tau)=\frac{12^{3} g_{2}^{3}(\tau)}{g_{2}^{3}(\tau)-27 g_{3}^{2}(\tau)}=1728+\frac{6^{6} g_{3}^{2}(\tau)}{g_{2}^{3}(\tau)-27 g_{3}^{2}(\tau)}
$$

for the $j$-invariant of the elliptic curve defined over $\mathbb{C}$ by

$$
y^{2}=4 x^{3}-g_{2} x-g_{3} .
$$




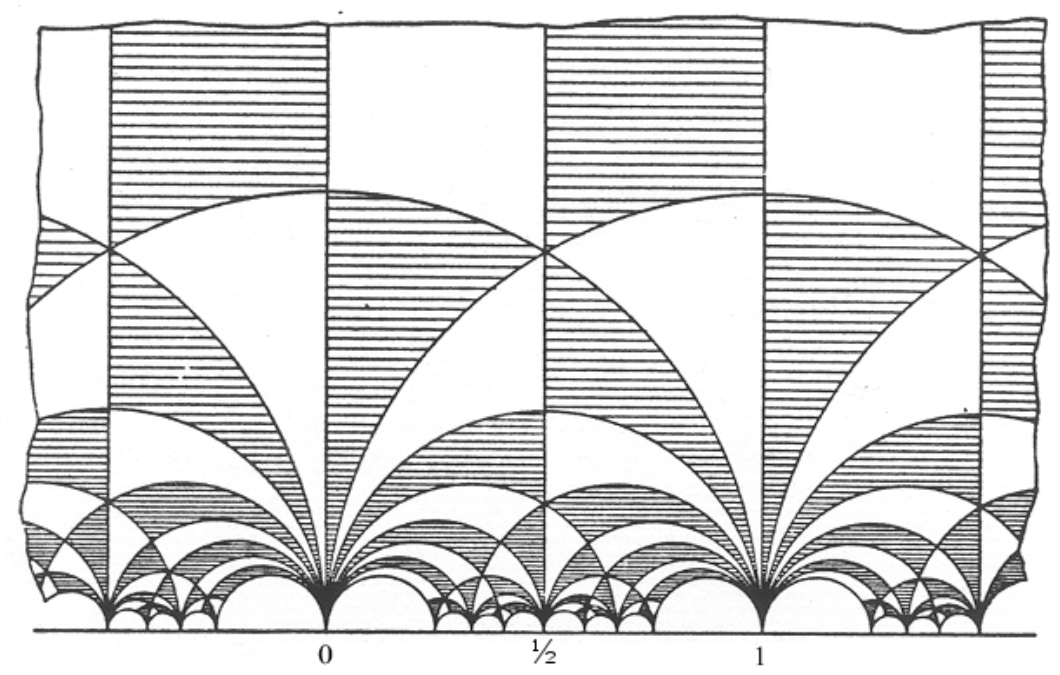

Figure 3 . Tessellation of $\mathcal{H}$ by fundamental domains for $\Gamma(1)$.

Here $g_{2}$ and $g_{3}$ are the Eisenstein series

$$
g_{2}(\tau)=60 \sum_{m, n}^{\prime}(m \tau+n)^{-4} \text { and } g_{3}(\tau)=140 \sum_{m, n}^{\prime}(m \tau+n)^{-6},
$$

the primed sums being over all nonzero integer pairs $(m, n)$. It follows from the basic theory of elliptic functions that

$$
\Delta(\tau)=g_{2}^{3}(\tau)-27 g_{3}^{2}(\tau)=(2 \pi)^{12} q \prod_{n \geq 1}\left(1-q^{n}\right)^{24}
$$

and that $j$ is a modular function for $\Gamma(1)$ that is holomorphic on $\mathcal{H}$ with $q$-expansion

$$
j(\tau)=\frac{\left(1+240 \sum_{m, n \geq 1} m^{3} q^{m n}\right)^{3}}{q \prod_{n \geq 1}\left(1-q^{n}\right)^{24}}=q^{-1}+744+\cdots .
$$

The Fourier coefficients are integers with some remarkable interpretations $\mathrm{Bor}$. It is easily seen that $g_{2}(\rho)=g_{3}(i)=0$ and so from (6.1) we have that $j(\rho)=0$ and $j(i)=1728$.

The Rogers-Ramanujan continued fraction $r$ is a modular function for $\Gamma(5)$. By (4.1), $r(\tau)$ is analytic in $\mathcal{H}$ and has no zeros there. By Proposition 2 we have that $r$ transforms correctly, and one checks that the inequivalent cusps of $\Gamma(5)$ are exactly the 10 rational numbers $b / d$ in the product (5.1) together with $i \infty$ and $-\frac{7}{5}$. Each cusp has width 5 , which is also the number of fundamental regions for $\Gamma(1)$ coming into each cusp. The $q$-expansion of $r(\tau)$ comes from (4.1)

$$
r(\tau)=q^{1 / 5}\left(1-q+q^{2}-q^{4}+q^{5}-q^{6}+q^{7}-q^{9}+2 q^{10}-3 q^{11}+\cdots\right)
$$




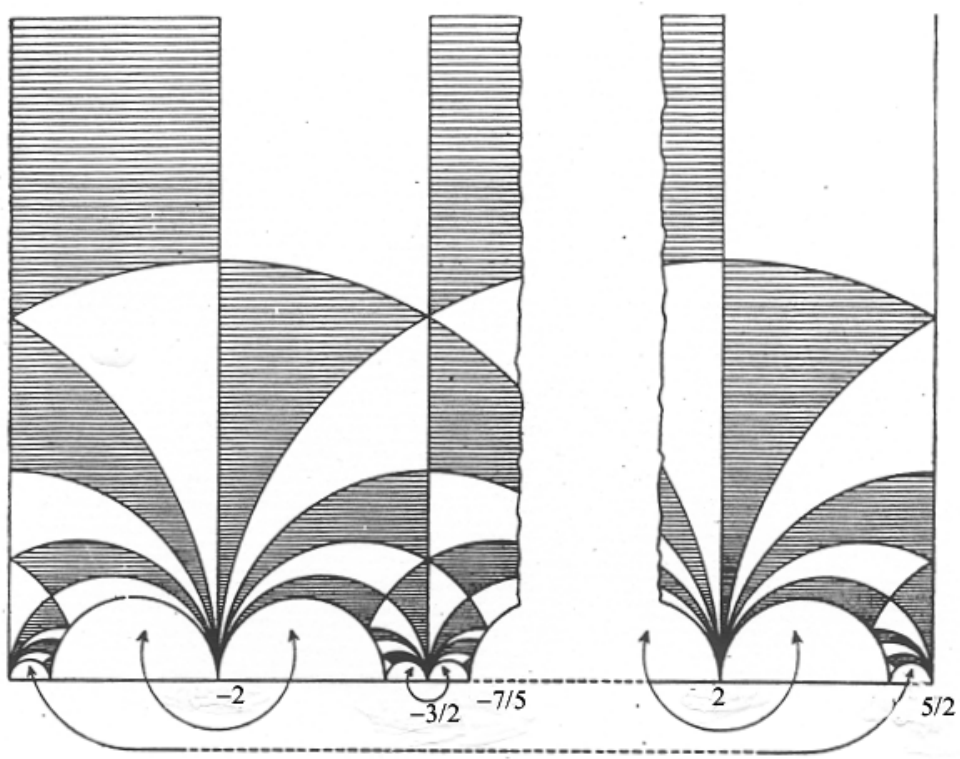

Figure 4. Fundamental domain for $\Gamma(5)$.

and has integer coefficients. The values of $r$ at the other cusps of $\Gamma(5)$ correspond precisely to the 11 nonzero vertices of the icosahedron. Therefore we see that $r$ is holomorphic at all except for $-\frac{7}{5}$, where by (2.6) and (4.1) again it has the Laurent expansion

$$
r\left(\frac{-7 \tau-10}{5 \tau+7}\right)=\frac{-1}{r(\tau)}=-q^{-1 / 5}\left(1+q-q^{3}+q^{5}+q^{6}-q^{7}-2 q^{8}+2 q^{10}+\cdots\right) .
$$

The coefficients of both expansions (6.4) and (6.5) have an interesting combinatorial meaning (see [An1] and [iir]). Figure 4 shows a (partial) picture of a fundamental domain for $\Gamma(5)$, including the cusps $-2,-\frac{3}{2},-\frac{7}{5}, 2$ and $\frac{5}{2}$. Figure 2 can now be interpreted as the image of this fundamental domain under the map $\tau \mapsto r(\tau)$. For instance, one may identify the point $\frac{-7+i}{5}$ in Figure 4 and its image $r\left(\frac{-7+i}{5}\right)=-i$ in Figure 2

If the (compact) Riemann surface from $\Gamma$ has genus 0 , then there is a Hauptmodul, a modular function with exactly one simple pole. A Hauptmodul will have exactly one simple zero and is uniquely determined by the location of its pole, its zero, and its (nonzero) value at another prescribed point. Any modular function for $\Gamma$ is a rational function of a Hauptmodul. The compact Riemann surface for $G=\Gamma(N)$ has genus 0 for $N \leq 5$ but not for $N>5$. We have seen that $j$ is a Hauptmodul for $\Gamma(1)$ and $r$ is one for $\Gamma(5)$. Hence $j$ can be be expressed as a rational function of $r$. In fact, the icosahedral invariant $J$ given in (5.3) provides this rational function for us. 
By Proposition 2, it is clear that the function on $\mathcal{H}$ defined by $J(r(\tau))$ satisfies $J(r(g \tau))=J(r(\tau))$ for all $g \in \Gamma(1)$. By what we know about the behavior of $r$ in the cusps, $V(r(\tau))$ never vanishes on $\mathcal{H}$. Hence $J(r(\tau))$ is holomorphic on $\mathcal{H}$, and it is also easy to check from (5.3) that it has a simple pole at $\infty$. Similarly, it must satisfy $J(r(\rho))=0$ and $J(r(i))=1728$. It follows that $J(r(\tau))=j(\tau)$ and hence that $r$ satisfies the icosahedral equation (2.5). In addition, we may conclude the following result from Proposition 4

Proposition 5. Assume that $\tau \in \mathcal{H}$ is such that $j(\tau) \neq 0,1728$. Then $\mathbb{Q}(\zeta, r(\tau))$ defines a Galois extension of $\mathbb{Q}(\zeta, j(\tau))$ whose Galois group $G$ is a subgroup of $\Gamma(1) / \Gamma(5) \simeq A_{5}$, proper if and only if (2.5) is reducible over $\mathbb{Q}(\zeta, j(\tau))$.

Theorem $\square$ is a consequence of Proposition $\left[5\right.$, since $A_{5}$ is not solvable but any proper subgroup of it is.

\section{The Dedekind eta-Function And $r(\tau)$}

Before turning to the proof of Theorem 2 I will first indicate how some of Ramanujan's well-known identitie ${ }^{7}$ involving $r(\tau)$ can be proven using the basic theory of modular functions for various congruence subgroups. Most of these identities involve the Dedekind eta-function

$$
\eta(\tau)=q^{1 / 24} \prod_{n \geq 1}\left(1-q^{n}\right),
$$

which transforms under the full modular group, as can be deduced from Euler's identity, itself a consequence of (4.8):

$$
\eta(\tau)=e\left(-\frac{1}{12}\right) \theta\left[\begin{array}{c}
1 / 3 \\
1
\end{array}\right](3 \tau) .
$$

The following explicit transformation law follows from [Kno, Thm. 2, p. 51]. For $\left(\begin{array}{l}a b \\ c d\end{array}\right) \in \mathrm{SL}(2, \mathbb{Z})$ with $c \geq 0$ and $d=1$ if $c=0$,

$$
\eta\left(\frac{a \tau+b}{c \tau+d}\right)=\nu_{\eta} \sqrt{c \tau+d} \eta(\tau)
$$

where

$$
\nu_{\eta}= \begin{cases}\left(\frac{d}{c}\right) e\left(\frac{(a+d) c-b d\left(c^{2}-1\right)-3 c}{24}\right) & \text { if } c \text { is odd } \\ \left(\frac{c}{|d|}\right) e\left(\frac{(a+d) c-b d\left(c^{2}-1\right)+3 d-3-3 c d}{24}\right) & \text { if } c \text { is even. }\end{cases}
$$

Here $(\div)$ is the Jacobi symbol with the convention that $\left(\frac{0}{1}\right)=1$.

Using (7.1) one may check that $\frac{\eta(\tau / 5)}{\eta(5 \tau)}$ is a modular function for $\Gamma(5)$. Since $r(\tau)$ is a Hauptmodul for $\Gamma(5)$, it is then not difficult to arrive at the following identity of Ramanujan:

$$
r^{-1}(\tau)-1-r(\tau)=\frac{\eta(\tau / 5)}{\eta(5 \tau)}
$$

used in Wa1 to prove (2.3).

An important congruence subgroup of level $N$ is

$$
\Gamma_{1}(N)=\left\{ \pm\left(\begin{array}{ll}
a & b \\
c & d
\end{array}\right) \in \Gamma(1): c \equiv 0(\bmod N) \text { and } d \equiv 1(\bmod N)\right\}
$$

\footnotetext{
${ }^{7} \mathrm{~A}$ proof of (7.2) below was first published in [Dar, of (7.3) in [Ra1], of (7.4) in [Ro2], of (7.5) in [Wa2], of [7.7] in [Dar] and [Ro2], and of (7.10) in [Dar]. Each identity occurs in Ramanujan's writings: (7.2) and (7.7) in Ram3 pp. 238, 239], (7.3) in [Ram3, p. 364], 7.4) and (7.5) in Ram1 pp. xxvii, xxviii] and (7.10) in Ram2] (see [Be, III, p. 257])
} 
It is well known that the genus of the associated compact Riemann surface is 0 exactly for $N \leq 10$ and for $N=12$ [Ogg, p. 109]. We are interested here in the example $\Gamma_{1}(5)$, which has inequivalent cusps at $i \infty, 0, \frac{1}{2}$ and $\frac{2}{5}$ with widths $1,5,5,1$, respectively. Using (4.9) and Lemma A.1 of the Appendix, it can be shown that $r^{5}(\tau)$ is a Hauptmodul for $\Gamma_{1}(5)$. Since $\Gamma_{1}(5)$ is normalized by the Fricke involution $\tau \mapsto \frac{-1}{5 \tau}$, we have that $r^{5}\left(\frac{-1}{5 \tau}\right)$ is a modular function for $\Gamma_{1}(5)$. We easily deduce the following identity:

$$
r^{5}\left(\frac{-1}{5 \tau}\right)=-\frac{r^{5}(\tau)-\varepsilon^{5}}{\varepsilon^{5} r^{5}(\tau)+1}, \quad \text { where } \varepsilon=\frac{-1+\sqrt{5}}{2} .
$$

This will be used in our proof of Theorem 2 given in the next section. Combining (7.3) with (3.2) we get the pretty identity

$$
\frac{r^{5}(\tau / 5)}{r(5 \tau)}=\frac{r^{4}(\tau)-3 r^{3}(\tau)+4 r^{2}(\tau)-2 r(\tau)+1}{r^{4}(\tau)+2 r^{3}(\tau)+4 r^{2}(\tau)+3 r(\tau)+1}
$$

Ramanujan stated in his first letter to Hardy. We may also derive the evaluation he gave in his second letter,

$$
r(\sqrt{-5})=\frac{\sqrt{5}}{1+\sqrt[5]{5^{3 / 4}((\sqrt{5}-1) / 2)^{5 / 2}-1}}-\frac{\sqrt{5}+1}{2},
$$

by an easy fixed point calculation.

Another important congruence subgroup of level $N$ is

$$
\Gamma_{0}(N)=\left\{ \pm\left(\begin{array}{ll}
a & b \\
c & d
\end{array}\right) \in \Gamma(1): c \equiv 0(\bmod N)\right\} .
$$

The associated compact Riemann surface has genus 0 exactly when $N \leq 10$ and $N=12,13,16,18,25$ Sc, Thm. 15, p. 103]. The group $\Gamma_{0}(5)$ has only two cusps, at $i \infty$ and 0 . It can be shown using (7.1) that a Hauptmodul for $\Gamma_{0}(5)$ is given by $(\eta(\tau) / \eta(5 \tau))^{6}$. A calculation using (4.9) and A.3) shows that for $\pm\left(\begin{array}{c}a b \\ c d\end{array}\right) \in \Gamma_{0}(5)$

$$
r^{5}\left(\frac{a \tau+b}{c \tau+d}\right)=\left(\frac{5}{d}\right)\left(r^{5}(\tau)\right)^{\left(\frac{5}{d}\right)}
$$

We can then easily derive the companion of (7.2):

$$
r^{-5}(\tau)-11-r^{5}(\tau)=\left(\frac{\eta(\tau)}{\eta(5 \tau)}\right)^{6} .
$$

It follows from (17.6) that the function defined by

$$
f(\tau)=\frac{1}{2 \pi i} \frac{d}{d \tau} \log \left(r^{5}(\tau)\right)
$$

transforms under $\pm\left(\begin{array}{l}a b \\ c d\end{array}\right) \in \Gamma_{0}(5)$ by

$$
f\left(\frac{a \tau+b}{c \tau+d}\right)=\left(\frac{5}{d}\right)(c \tau+d)^{2} f(\tau) .
$$

The function $f$ is an example of a modular form of weight 2 for $\Gamma_{0}(5)$ with character $\left(\frac{5}{2}\right)$. By (7.1) the function $\eta^{5}(5 \tau) / \eta(\tau)$ transforms in precisely the same way and it is readily seen that

$$
f(\tau) \frac{\eta(\tau)}{\eta^{5}(5 \tau)}=\left(\frac{\eta(\tau)}{\eta(5 \tau)}\right)^{6} .
$$

Combining (7.8), (7.9) and (4.1) we get 


$$
\frac{5}{2 \pi i} \frac{r^{\prime}(\tau)}{r(\tau)}=1-5 \sum_{n \geq 1}\left(\frac{5}{n}\right) \frac{n q^{n}}{1-q^{n}}=\frac{\eta^{5}(\tau)}{\eta(5 \tau)} .
$$

We can write this identity in the equivalent form

$$
r(\tau)=\frac{\sqrt{5}-1}{2} e\left(\frac{1}{5} \int_{0}^{\tau} \frac{\eta^{5}(z)}{\eta(5 z)} d z\right)
$$

upon using (2.2).

The series from (7.10)

$$
1-5 \sum_{n \geq 1}\left(\frac{5}{n}\right) \frac{n q^{n}}{1-q^{n}}=1-5 \sum_{n \geq 1}\left(\sum_{d \mid n}\left(\frac{5}{d}\right) d\right) q^{n}
$$

gives the $q$-expansion of an Eisenstein series of weight 2. There are a number of other identities connecting $r(\tau)$ with Eisenstein series that are amenable to a similar treatment using modular functions that we will not prove here. The following example, which appears in [Ram3], was proven in [An1, p. 102] by means of Ramanujan's " $\psi_{1}$-summation":

$$
r(\tau)^{3}=q^{3 / 5} \frac{\sum_{n \in \mathbb{Z}} \frac{q^{2 n}}{1-q^{5 n+2}}}{\sum_{n \in \mathbb{Z}} \frac{q^{n}}{1-q^{5 n+1}}} .
$$

This identity may also be written in the form

$$
r(\tau)^{3}=q^{3 / 5} \frac{\sum_{n=0}^{\infty} \frac{1+q^{5 n+2}}{1-q^{5 n+2}} q^{5 n^{2}+4 n}-\sum_{n=0}^{\infty} \frac{1+q^{5 n+3}}{1-q^{5 n+3}} q^{5 n^{2}+6 n+1}}{\sum_{n=0}^{\infty} \frac{1+q^{5 n+1}}{1-q^{5 n+1}} q^{5 n^{2}+2 n}-\sum_{n=0}^{\infty} \frac{1+q^{5 n+4}}{1-q^{5 n+4}} q^{5 n^{2}+8 n+3}} .
$$

See An2 for more examples of this type.

\section{Modular Curves ANd COMPlex Multiplication}

The compact Riemann surfaces associated to $\Gamma(N), \Gamma_{1}(N)$ and $\Gamma_{0}(N)$ are the complex points of algebraic curves $X(N), X_{1}(N)$ and $X_{0}(N)$, known as modular curves, that can even be defined over $\mathbb{Q}$ Shi]. Furthermore, their non-cusp points may be interpreted as parameterizing elliptic curves with various level- $N$ structures; i.e. modular curves are moduli spaces for elliptic curves.

In particular, the non-cusp points of $X_{1}(N)$ parameterize elliptic curves together with a rational point of order $N$. For $N=5$, for instance, we have an explicit equation for such a curve $E$ (Tate's form):

$$
y^{2}+(1-b) x y-b y=x^{3}-b x^{2} .
$$

One may check that $P=(0,0)$ is a point of order 5 on $E$, with $2 P=\left(b, b^{2}\right), 3 P=$ $(b, 0), 4 P=(0, b), 5 P=0$. This curve has $j$-invariant

$$
j(E)=\frac{\left(b^{4}-12 b^{3}+14 b^{2}+12 b+1\right)^{3}}{b^{5}\left(b^{2}-11 b-1\right)} .
$$

Upon making the substitution $b=b(\tau)=-r^{5}\left(\frac{-1}{5 \tau}\right)=\frac{r^{5}(\tau)-\varepsilon^{5}}{\varepsilon^{5} r^{5}(\tau)+1}$ from (7.3), a (machine) calculation shows that

$$
j(E)=\frac{-\left(r^{20}(\tau)-228 r^{15}(\tau)+494 r^{10}(\tau)+228 r^{5}(\tau)+1\right)^{3}}{r^{5}(\tau)\left(r^{10}(\tau)+11 r^{5}(\tau)-1\right)^{5}},
$$

which, in view of the icosahedral equation (2.5), shows that $j(E)=j(\tau)$. Thus the Hauptmodul $b(\tau)=-r^{5}\left(\frac{-1}{5 \tau}\right)$ for $\Gamma_{1}(5)$ determines a curve $E$ from (8.1) with $j$-invariant $j(\tau)$. 
If we assume that $j(\tau) \neq 0,1728$ is algebraic, then $E$ is isomorphic over $\overline{\mathbb{Q}}$ to the curve $E_{\tau}$ defined over the number field $\mathbb{Q}(j(\tau))$ by

$$
y^{2}=4 x^{3}+c x+c \quad \text { where } c=\frac{27 j(\tau)}{1728-j(\tau)},
$$

since $j\left(E_{\tau}\right)=j(\tau)$. In general let $E_{\tau}[N]$ denote the group of $N$-torsion points in $E_{\tau}(\overline{\mathbb{Q}})$ and $\mathbb{Q}\left(j(\tau), E_{\tau}[N]\right)$ denote the $N$-division field of $E_{\tau}$, obtained by adjoining to $\mathbb{Q}(j(\tau))$ both coordinates of all points of $E_{\tau}[N]$. Using the basic facts about this field (see [Shi, p. 135]), one can readily show that $b(\tau)$ is contained in $\mathbb{Q}\left(j(\tau), E_{\tau}[5]\right)$.

We may now give a rather direct proof of Theorem 2 of \&2. If $\tau$ is in an imaginary quadratic field $\mathbb{K}$, then $E_{\tau}$ has extra endomorphisms ("complex multiplications") forming an order $\mathcal{O}$ in $\mathbb{K}$. It is a consequence of the First Main Theorem of complex multiplication that $j(\tau)$ is an algebraic integer and that $\mathbb{K}(j(\tau))$ is an abelian extension of $\mathbb{K}$ whose Galois group is isomorphic to the group of (proper) ideal classes of $\mathcal{O}\left[\mathrm{Cox}\right.$ Thm. 11.1, p. 220]. Furthermore, $\mathbb{K}\left(j(\tau), E_{\tau}[N]\right)$ is an abelian extension of $\mathbb{K}(j(\tau))$ [Sil, Thm. 2.3, p. 108]. In particular, $b(\tau) \in \mathbb{Q}\left(j(\tau), E_{\tau}[5]\right)$ is expressible in terms of radicals over $\mathbb{Q}$ and hence so is $r(\tau)$ since $b(\tau)=\frac{r^{5}(\tau)-\varepsilon^{5}}{\varepsilon^{5} r^{5}(\tau)+1}$. This proves Theorem 2 .

Turning now to the proof of Theorem 3 of $\$ 2$, we have the classical result of Schneider [S] stating that if $j(\tau)$ is algebraic for an algebraic $\tau$, then $\tau$ must be imaginary quadratic. The proof of Theorem 3 comes down to the (tedious) task of factoring (2.5) for each of the 13 integer values of $j(\tau)$ and each of the 29 quadratic values (up to Galois conjugation) of $j(\tau)$ that occur for imaginary quadratic values of $\tau$ and are listed in [Ber]. We must be sure that this list is complete. By Cox. Thm. 10.23, p. 214], we are reduced to finding all imaginary quadratic orders with class number $\leq 2$. By the class number formula [Cox, p. 146] it is enough to find all such maximal orders, and these have all been determined (see Sta] and its references).

In general, suppose that $f$ is any modular function for $\Gamma(N)$ whose Fourier coefficients are contained in $\mathbb{Q}\left(\zeta_{N}\right)$. The set of all such modular functions form a field. It is shown in Shi, Prop. 6.9(1), p. 140] that this field is generated over $\mathbb{Q}$ by $j$ and the $N^{2}-1$ distinct modular functions for $\Gamma(N)$ given by

$$
f_{\alpha}(\tau)=\frac{g_{2}(\tau) g_{3}(\tau)}{\Delta(\tau)}\left(\frac{1}{\left(\alpha_{1}+\alpha_{2} \tau\right)^{2}}+\sum_{m, n}^{\prime}\left(\frac{1}{\left(\alpha_{1}+\alpha_{2} \tau-m-n \tau\right)^{2}}-\frac{1}{(m+n \tau)^{2}}\right)\right),
$$

where $\alpha=\left(\alpha_{1}, \alpha_{2}\right) \in \mathbb{Q}^{2}$ with $\alpha \notin \mathbb{Z}^{2}$, has $N \alpha \in \mathbb{Z}^{2}$. Here $g_{2}$ and $g_{3}$ and $\Delta$ are defined in (6.2) and (6.3). Observe that $f_{\alpha}(\tau)$ is constructed from the Weierstrass $\wp-$ function for the lattice $\mathbb{Z} \oplus \tau \mathbb{Z}$ specialized at an $N$-torsion point. For later purposes set $f_{(1,0)}=0$.

Suppose that $\tau \in \mathcal{H}$ is not a pole of $f$ and is such that $j(\tau) \neq 0,1728$ is algebraic (these omitted cases may be handled separately). It follows easily that $f(\tau)$ is in fact contained in the $N$-division field $\mathbb{Q}\left(j(\tau), E_{\tau}[N]\right)$ of $E_{\tau}$. Also, the same argument as before shows that this field is solvable over $\mathbb{Q}$ if $\tau$ is in an imaginary quadratic field $\mathbb{K}$. A deeper consequence of the theory of complex multiplication in this case is that $\mathbb{K}(j(\tau), f(\tau))$ is an abelian extension of $\mathbb{K}$. Suppose that $\mathbb{K}=\mathbb{Q}(\sqrt{D})$ has discriminant $D<-4$. The Second Main Theorem of complex multiplication (for elliptic curves) implies the converse statement, that every abelian extension of $\mathbb{K}$ is contained in $\mathbb{K}\left(j(\tau), f_{(1 / N, 0)}(\tau)\right)$ for $\tau=\frac{D+\sqrt{D}}{2}$ and some $N \in \mathbb{Z}^{+}[\overline{\mathrm{Deu}}$, $\S 24]$. See [BCHIS, I] and [Cox, p. 242] for more information and further references. 


\section{More COntinued Fractions}

There are several other continued fractions that occur in the theory of modular functions. Ramanujan studied the following two relatives of $r(\tau)$ :

$$
\begin{aligned}
& u(\tau)=\frac{\sqrt{2} q^{1 / 8}}{1+} \frac{q}{1+q+} \frac{q^{2}}{1+q^{2}+} \frac{q^{3}}{1+q^{3}+} \frac{q^{4}}{1+q^{4}+} \ldots \\
& \mathrm{v}(\tau)=\frac{q^{1 / 2}}{1+q+} \frac{q^{2}}{1+q^{3}+} \frac{q^{4}}{1+q^{5}+} \frac{q^{6}}{1+q^{7}+} \frac{q^{8}}{1+q^{9}+} \ldots,
\end{aligned}
$$

which appear in the first two entries of Chapter XIX of his second notebook Ram2 p. 229]. His assertions there can be put in the form of the following analogues of Rogers' identity (4.1):

$$
\begin{array}{r}
u(\tau)=\sqrt{2} q^{1 / 8} \prod_{n \geq 1}\left(1+q^{n}\right)^{(-1)^{n}} \\
\mathrm{v}(\tau)=q^{1 / 2} \prod_{n \geq 1}\left(1-q^{n}\right)^{\left(\frac{8}{n}\right),}
\end{array}
$$

for $\tau \in \mathcal{H}$. Proofs of (9.4) were first published in [Göl] and [Gor]. It seems to have not been observed in print that (9.3) follows immediately from an identity first stated by Eisenstein in 1844 [Eis, p. 290 in Werke]:

$$
\prod_{n \geq 0} \frac{1-x q^{n}}{1-y q^{n}}=\frac{1}{1+} \frac{x-y}{1-q+} \frac{q y-x}{1+q+} \frac{q^{2} x-q y}{1-q^{3}+} \frac{q^{3} y-q x}{1+q^{2}+} \frac{q^{4} x-q^{2} y}{1-q^{5}+} \ldots
$$

valid when $\tau \in \mathcal{H}$ and $|y|<1$. Eisenstein gave no indication of how he proved (9.5), and the proof published in 1877 by Muir [Mu2, which refers to [Mu1], appears to be incomplete. See [Fol] for a proof and a discussion of Eisenstein's other continued fractions.

The special values of these continued fractions are also interesting and even simpler than those of $r(\tau)$. We next establish the following identities connecting these continued fractions with each other and with $j$ :

$$
\begin{array}{r}
\left(u^{16}+14 u^{8}+1\right)^{3}-2^{-4} j(\tau) u^{8}\left(u^{8}-1\right)^{4}=0 \\
u^{4}\left(\mathrm{v}^{2}+1\right)^{2}+4 \mathrm{v}\left(\mathrm{v}^{2}-1\right)=0 .
\end{array}
$$

These equations are both solvable over $\mathbb{Q}(j(\tau))$ and if $\tau$ is in an imaginary quadratic field, then $u(\tau)$ and $\mathrm{v}(\tau)$ can be expressed in terms of radicals over $\mathbb{Q}$, and $\mathrm{v}(\tau)$ is a unit.

To prove (9.6), from (9.3) we can write

$$
u(\tau)=\sqrt{2} q^{1 / 8} \prod_{n \geq 1} \frac{\left(1-q^{2 n-1}\right)}{\left(1-q^{4 n-2}\right)^{2}}=\sqrt{2} \frac{\eta(\tau) \eta^{2}(4 \tau)}{\eta^{3}(2 \tau)}
$$

and a calculation using the product formula (4.8) gives

$$
u^{2}(\tau)=2 \frac{\eta^{2}(\tau) \eta^{4}(4 \tau)}{\eta^{6}(2 \tau)}=\theta\left[\begin{array}{l}
1 \\
0
\end{array}\right](2 \tau) / \theta\left[\begin{array}{l}
0 \\
0
\end{array}\right](2 \tau) .
$$

One may check using (4.7) and (4.4) that

$$
u^{2}(2(\tau+1))=i u^{2}(2 \tau) \text { and } u^{2}\left(2\left(\frac{-1}{\tau}\right)\right)=\frac{1-u^{2}(2 \tau)}{1+u^{2}(2 \tau)} .
$$

These two transformations generate the octahedral group, which is the image of the symmetry group of the regular octahedron stereographically projected on the 
complex plane, as in the case of the icosahedron. Proceeding as we did with $r$, one derives (9.6), which is Klein's octahedral equation evaluated at $u^{2}$. The fact that (9.6) is solvable over $\mathbb{Q}(j(\tau))$ follows from [Dic p. 238]. Since $u^{2}$ has its only pole at the cusp $\frac{1}{2}$ with Laurent expansion there

$$
u^{2}\left(\frac{\tau}{2 \tau+1}\right)=\frac{2}{u^{2}(\tau)}=2 q^{-1 / 4}(1-2 q+\ldots),
$$

it follows that $u^{2}$ is a Hauptmodul for $\Gamma(4)$.

In order to derive (9.7) we need to consider the congruence subgroup $\Gamma_{1}(8)$. The Riemann surface associated to $\Gamma_{1}(8)$ has genus 0 and cusps at $i \infty, 0, \frac{1}{4}, \frac{1}{3}, \frac{3}{8}$, and $\frac{1}{2}$ with widths $1,8,2,8,1$ and 4 , respectively. It can be shown using

$$
\mathrm{v}(\tau)=e\left(-\frac{1}{8}\right) \frac{\theta\left[\begin{array}{c}
3 / 4 \\
1
\end{array}\right](8 \tau)}{\theta\left[\begin{array}{c}
1 / 4 \\
1
\end{array}\right](8 \tau)}
$$

and Lemma A.1 that $\mathrm{v}^{2}$ is a Hauptmodul for $\Gamma_{1}(8)$ with its zero at $\infty$ and its pole at $\frac{3}{8}$. Furthermore, we have that

$$
\mathrm{v}^{2}(0)=3-2 \sqrt{2}, \quad \mathrm{v}^{2}\left(\frac{1}{4}\right)=1, \quad \mathrm{v}^{2}\left(\frac{1}{3}\right)=3+2 \sqrt{2} \quad \text { and } \quad \mathrm{v}^{2}\left(\frac{1}{2}\right)=-1 .
$$

Now $u^{8}$ is a modular function for $\Gamma_{1}(8)$ with simple zeros at $\infty$ and $\frac{3}{8}$ and a double zero at $\frac{1}{4}$ and no others, and a pole of order 4 at $\frac{1}{2}$ and no others. Since $\frac{16 \mathrm{v}^{2}\left(1-\mathrm{v}^{2}\right)^{2}}{\left(1+\mathrm{v}^{2}\right)^{4}}$ has the same zeros and poles, the quotient of these two modular functions is an entire function on a compact Riemann surface, hence constant. It is then easy to see that this constant must be 1 and that (9.7) follows by taking the correct square root.

One can also readily derive the following analogues of (7.3) and (7.7) by using $\Gamma_{1}(8)$ and $\Gamma_{0}(8)$ :

$$
\mathrm{v}^{2}\left(\frac{-1}{8 \tau}\right)=\frac{\mathrm{v}^{2}(\tau)-\sigma^{2}}{\sigma^{2} \mathrm{v}^{2}(\tau)-1} \quad \text { and } \quad \mathrm{v}^{-2}(\tau)+\mathrm{v}^{2}(\tau)-6=\frac{\eta^{4}(\tau) \eta^{2}(4 \tau)}{\eta^{2}(2 \tau) \eta^{4}(8 \tau)}
$$

where $\sigma=-1+\sqrt{2}$.

In addition to proving (9.3), Eisenstein's identity (9.5) also provides other continued fractions apparently not in Ramanujan's writings. For example, let

$$
w(\tau)=\frac{\sqrt{2} q^{1 / 24}}{1-} \frac{q}{1-q+1} \frac{q}{1+q-} \frac{q^{3}}{1-q^{3}+} \frac{q^{2}}{1+q^{2}-} \frac{q^{5}}{1-q^{5}+} \frac{q^{3}}{1+q^{3}-} \ldots,
$$

where the pattern is easily discerned by looking at every other term. Then it follows from (9.5) that for $\tau \in \mathcal{H}$,

$$
w(\tau)=\sqrt{2} q^{1 / 24} \prod_{n \geq 1}\left(1+q^{n}\right) .
$$

Actually, $w$ as defined by the product (9.9) is one of the classical Weber functions, denoted by him $f_{2}$, that can be expressed in terms of the $\eta$-function by

$$
w(\tau)=\sqrt{2} \eta(2 \tau) / \eta(\tau) .
$$

Using (7.1) we can show that $w^{24}$ is a Hauptmodul for $\Gamma_{1}(2)=\Gamma_{0}(2)$ and from this arrive at Weber's Web identity:

$$
\left(w^{24}+16\right)^{3}-j(\tau) w^{24}=0,
$$

which is obviously solvable over $\mathbb{Q}(j(\tau))$. Also, we have from (9.10) and (9.8) that

$$
u(\tau)=w^{2}(2 \tau) / w(\tau) .
$$


Weber [Web, pp. 457-499] obtained many results about the special values of $w$ and of the related products

$$
q^{-1 / 48} \prod_{n \geq 1}\left(1+q^{n-1 / 2}\right) \quad \text { and } q^{-1 / 48} \prod_{n \geq 1}\left(1-q^{n-1 / 2}\right),
$$

which clearly both have continued fraction expansions derivable from (9.5). Ramanujan also explicitly evaluated these products for a large number of imaginary quadratic values of $\tau$ (see e.g. Ram1, \#6]).

It is not obvious from (9.6) or (9.11) whether or not $u(\tau)$ or $w(\tau)$ is a unit when $\tau$ is in an imaginary quadratic field, although it is clear that the only troublesome prime is 2. If, for example, $\tau=\sqrt{-m}$ where $m$ is a positive integer with $m \equiv$ $1(\bmod 4)$, then $2^{-1 / 8} w(\tau)$ is a unit [Bir, p. 290]. It follows easily from (9.11) that $2^{6} \mid j(\tau)$, and so from (9.6) we have that $u(\tau)$ is also a unit in this case. Similar results for other $\tau$ can also be given by using results from $\mathrm{Bir}$ in combination with the identity (9.12).

Finally, we remark that there are other continued fraction expansions for $w, u$ and $\mathrm{v}$, originally established as identities for the corresponding infinite products. The following continued fraction for $w$ as defined by the product 9.9) was given by Heine Hei] as early as 1846 by means of Euler's transformation:

$$
w(\tau)=\sqrt{2} q^{1 / 24}\left(1+\frac{q}{1-q+} \frac{q^{3}-q^{2}}{1+} \frac{q^{5}-q^{3}}{1+} \frac{q^{7}-q^{4}}{1+} \ldots\right),
$$

for $\tau \in \mathcal{H}$. In his first paper in 1937, Selberg [Se1] provided the following continued fractions for the products in (9.3) and (9.4):

$$
u(\tau)=\frac{\sqrt{2} q^{1 / 8}}{1+} \frac{q}{1+} \frac{q+q^{2}}{1+} \frac{q^{3}}{1+} \frac{q^{2}+q^{4}}{1+} \ldots \text { and } \mathrm{v}(\tau)=\frac{q^{1 / 2}}{1+} \frac{q+q^{2}}{1+} \frac{q^{4}}{1+} \frac{q^{3}+q^{6}}{1+} \frac{q^{8}}{1+} \ldots
$$

for $\tau \in \mathcal{H}$, and he also established that

$$
\frac{q^{1 / 3}}{1+} \frac{q+q^{2}}{1+} \frac{q^{2}+q^{4}}{1+} \frac{q^{3}+q^{6}}{1+} \ldots=q^{1 / 3} \prod_{n \geq 1} \frac{\left(1-q^{6 n-1}\right)\left(1-q^{6 n-5}\right)}{\left(1-q^{6 n-3}\right)^{2}} .
$$

Unknown to Selberg at the time, the last three identities all appear (without proof) in Ramanujan's "lost" notebook (see [An1]). We leave the treatment of the continued fraction (9.13) using $\Gamma(6)$ along the lines of this paper as a (challenging) exercise.

\section{BEyOND CONTINUED FRACTIONS}

In this final section I will briefly explore the simplest case when continued fractions are no longer adequate to represent the modular functions of interest. This happens for $\Gamma(7)$. However, some of the themes we have been developing persist, albeit in a more complicated form.

Define the quotient of two infinite determinants $\left|a_{i, j}\right|\left|b_{i, j}\right|^{-1}$ to be

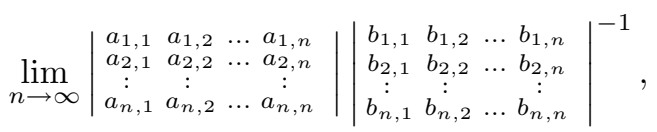

provided this limit exists. Using an idea going back to Sylvester [Syl, tri-diagonal determinants yield continued fractions:

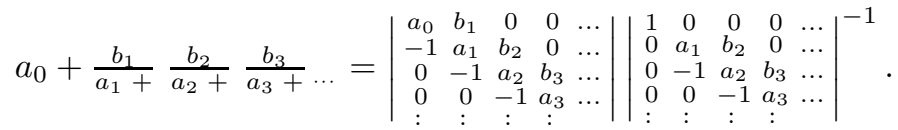


Thus we can express the fundamental unit in $\mathbb{Q}(\sqrt{5})$, the real quadratic field with the smallest discriminant 5 , as

$$
\frac{1+\sqrt{5}}{2}=\left|\begin{array}{ccccc|ccccc}
1 & 1 & 0 & 0 & \cdots \\
-1 & 1 & 1 & 0 & \cdots \\
0 & -1 & 1 & 1 & \cdots & 1 & 0 & 0 & 0 & \cdots \\
0 & 1 & 1 & 0 & \cdots \\
0 & -1 & 1 & 1 & \cdots \\
\vdots & \vdots & \vdots & \vdots & \cdots
\end{array}\right|^{-1} .
$$

A generalization to cubic number fields of the periodic continued fraction expansions of real quadratic units was first proposed by Jacobi Jac. Consider the number field $\mathbb{L}=\mathbb{Q}\left(\varepsilon_{1}\right) \subset \mathbb{Q}\left(\zeta_{7}\right)$, where

$$
\varepsilon_{1}=\frac{\sin (3 \pi / 7)}{\sin (\pi / 7)}
$$

satisfies the equation $x^{3}-2 x^{2}-x+1=0$. This is the real cubic number field with the smallest possible discriminant $7^{2}$. Now $\varepsilon_{1}$ is a unit (a cyclotomic unit) that may be expressed as a quotient of periodic infinite determinants:

$$
\varepsilon_{1}=\left|\begin{array}{cccccc|cccccc}
2 & 1 & -1 & 0 & 0 & \cdots \\
-1 & 2 & 1 & -1 & 0 & \cdots \\
0 & -1 & 2 & 1 & -1 & \cdots \\
0 & 0 & -1 & 2 & 1 & \cdots & 0 & 0 & 0 & 0 & \cdots \\
0 & 2 & 1 & -1 & 0 & \cdots \\
0 & -1 & 2 & 1 & -1 & \cdots \\
0 & 0 & -1 & 2 & 1 & \cdots \\
\vdots & \vdots & \vdots & \vdots & \vdots & \vdots
\end{array}\right|^{-1}
$$

Consider for $\tau \in \mathcal{H}$ the function

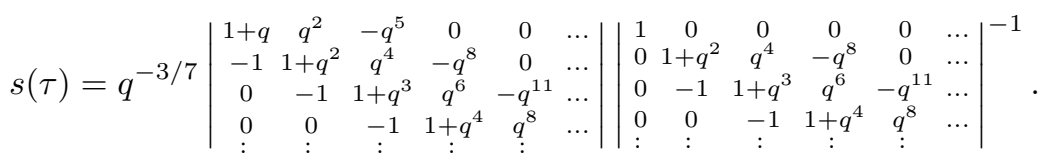

This quotient also converges for $\tau=0$, where it reduces to (10.1).

By now the reader has likely guessed that $s$ is a modular function, and in fact it is one for $\Gamma(7)$. It also has a nice relation with the elliptic curve $E$ in Tate's form:

$$
y^{2}+\left(1+d-d^{2}\right) x y+\left(d^{2}-d^{3}\right) y=x^{3}+\left(d^{2}-d^{3}\right) x^{2},
$$

for which $P=(0,0)$ is a point of order 7 . To see this, first consider the modular function

$$
h(\tau)=q^{-1} \prod_{n \geq 1} \frac{\left(1-q^{7 n-2}\right)^{2}\left(1-q^{7 n-5}\right)^{2}\left(1-q^{7 n-3}\right)\left(1-q^{7 n-4}\right)}{\left(1-q^{7 n-1}\right)^{3}\left(1-q^{7 n-6}\right)^{3}} .
$$

It can be shown that $h$ is a Hauptmodul for $\Gamma_{1}(7)$ that transforms under the Fricke involution by the formula

$$
h\left(\frac{-1}{7 \tau}\right)=\frac{h(\tau)-\varepsilon_{1}^{2} \varepsilon_{2}^{-3}}{\varepsilon_{1}^{-1} \varepsilon_{2}^{-2} h(\tau)-1},
$$

where $\varepsilon_{2}=\frac{\sin (2 \pi / 7)}{\sin (\pi / 7)}$. Here $\left\{\varepsilon_{1}, \varepsilon_{2}\right\}$ form a basis for the units of infinite order in $\mathbb{L}$. Now set $d(\tau)=h\left(\frac{-1}{7 \tau}\right)$. Then the elliptic curve $E$ of (10.2) with $d=d(\tau)$ has

$$
j(E)=j(\tau)=\frac{\left(d^{2}-d+1\right)^{3}\left(d^{6}-11 d^{5}+30 d^{4}-15 d^{3}-10 d^{2}+5 d+1\right)^{3}}{d^{7}(d-1)^{7}\left(d^{3}-8 d^{2}+5 d+1\right)} .
$$

Furthermore, the function $s(\tau)$ is related to $h(\tau)$ by the following identity:

$$
s^{7}=h(h-1)^{2},
$$

which may be taken as a defining equation for the genus 3 curve $X(7)$, the famous Klein curve. The special values of $s(\tau)$ for a given $j(\tau)$ can now be obtained in much the same way as those of $r(\tau)$ by solving (10.3) for $d$ and then finding $s$ from (10.4) when

$$
h=\frac{d-\varepsilon_{1}^{2} \varepsilon_{2}^{-3}}{\varepsilon_{1}^{-1} \varepsilon_{2}^{-2} d-1} .
$$


To see that $s(\tau)$ is a modular function, consider the determinant of width 4 :

$$
S(z)=\left|\begin{array}{cccccc}
1+q z & q^{2} z^{2} & -q^{5} z^{3} & 0 & 0 & \cdots \\
-1 & 1+q^{2} z & q^{4} z^{2} & -q^{8} z^{3} & 0 & \cdots \\
0 & -1 & 1+q^{3} z & q^{6} z^{2} & -q^{11} z^{3} & \cdots \\
0 & 0 & -1 & 1+q^{4} & q^{8} & \cdots \\
\vdots & \vdots & \vdots & \vdots & \vdots &
\end{array}\right|
$$

for $\tau \in \mathcal{H}$, so that

$$
s(\tau)=q^{-3 / 7} S(1) / S(q) .
$$

It is easily checked that

$$
S(z)=(1+q z) S(q z)+q^{2} z^{2} S\left(q^{2} z\right)-q^{5} z^{3} S\left(q^{3} z\right) .
$$

Selberg's paper [Se1] mentioned at the end of \$9 studies a generalization of the auxiliary function used in Rogers' proof of the Rogers-Ramanujan identities. This function is defined for $\tau \in \mathcal{H}$ and $k>-1 / 2$ by

$$
C_{k, j}(z)=1-z^{j} q^{j}+\sum_{n \geq 1}(-1)^{n} z^{k n} q^{\frac{1}{2}(2 k+1)\left(n^{2}+n\right)-j n}\left(1-z^{j} q^{(2 n+1) j}\right) \frac{(z q, q)_{n}}{(q, q)_{n}}
$$

where we use the standard notation

$$
(a, q)_{n}=(1-a)(1-a q) \cdots\left(1-a q^{n-1}\right) .
$$

Selberg showed that $C_{k, j}$ satisfies the recurrence formulas

$$
\begin{aligned}
C_{k,-j}(z) & =-z^{-j} q^{-j} C_{k, j}(z) \\
C_{k, j}(z) & =C_{k, j-1}(z)+z^{j-1} q^{j-1}(1-z q) C_{k, k-j+1}(z q)
\end{aligned}
$$

and from these derived functional relations for $C_{k, k}(z)$. In particular, it can be shown using Selberg's formulas and (10.5) that

$$
S(z)=\frac{C_{3,3}(z)}{\prod_{n \geq 1}\left(1-z q^{n}\right)} .
$$

Jacobi's triple product identity (4.8) then yields the identities

$$
\begin{aligned}
& \prod_{n \geq 1} \frac{\left(1-q^{7 n-3}\right)\left(1-q^{7 n-4}\right)\left(1-q^{7 n}\right)}{\left(1-q^{n}\right)}=S(1) \\
& \prod_{n \geq 1} \frac{\left(1-q^{7 n-2}\right)\left(1-q^{7 n-5}\right)\left(1-q^{7 n}\right)}{\left(1-q^{n}\right)}=S(1)-q^{2} S\left(q^{2}\right) \\
& \prod_{n \geq 1} \frac{\left(1-q^{7 n-1}\right)\left(1-q^{7 n-6}\right)\left(1-q^{7 n}\right)}{\left(1-q^{n}\right)}=S(q) .
\end{aligned}
$$

Thus we have

$$
s(\tau)=q^{-3 / 7} \prod_{n \geq 1} \frac{\left(1-q^{7 n-3}\right)\left(1-q^{7 n-4}\right)}{\left(1-q^{7 n-1}\right)\left(1-q^{7 n-6}\right)}=e\left(\frac{1}{7}\right) \frac{\theta\left[\begin{array}{c}
1 / 7 \\
1
\end{array}\right](7 \tau)}{\theta\left[\begin{array}{c}
5 / 7 \\
1
\end{array}\right](7 \tau)},
$$

which is seen to be a modular function for $\Gamma(7)$, as is

$$
t(\tau)=q^{-2 / 7} \prod_{n \geq 1} \frac{\left(1-q^{7 n-2}\right)\left(1-q^{7 n-5}\right)}{\left(1-q^{7 n-1}\right)\left(1-q^{7 n-6}\right)}=e\left(\frac{1}{14}\right) \frac{\theta\left[\begin{array}{c}
3 / 7 \\
1
\end{array}\right](7 \tau)}{\theta\left[\begin{array}{c}
5 / 7 \\
1
\end{array}\right](7 \tau)} .
$$

From the transformation law (4.5) of the theta constants under $g \in \Gamma(1)$ one shows that

$$
(s(g \tau), t(g \tau))=\left(\frac{a s(\tau)+b t(\tau)+c}{a^{\prime \prime} s(\tau)+b^{\prime \prime} t(\tau)+c^{\prime \prime}}, \frac{a^{\prime} s(\tau)+b^{\prime} t(\tau)+c^{\prime}}{a^{\prime \prime} s(\tau)+b^{\prime \prime} t(\tau)+c^{\prime \prime}}\right)
$$


defines a (projective) representation of $\Gamma(1)$ that gives an embedding of $\Gamma(1) / \Gamma(7)$ into $P S L(3, \mathbb{C})$. The corresponding invariant theory was also studied by Klein [Kl3]. For a detailed exposition of this and of the amazingly rich structure of the modular curve $X(7)$ see Elk. The identities (10.3) and 10.4) follow from this theory.

Much of this development, including Selberg's recurrence, the theta representation and the invariant theory, can be generalized to higher levels. We have already seen this for algebraic properties of the special values and the CM theory. The role of units invites further exploration.

\section{ACKNOWEDGEMENTS}

I thank Barry Mazur and J-P. Serre for their comments on an earlier version of this paper.

\section{Appendix A}

This appendix contains a technical result that is useful to verify transformation properties of modular functions that are expressed as the quotient of theta constants with the same integral value of the bottom characteristic $\epsilon^{\prime}$ and the same argument $N \tau$, for a positive integer $N$. It is convenient to let $\lambda$ denote some eighth root of unity that does not depend on either $\epsilon$ or $\tau$ but whose value may differ in different expressions.

Lemma A.1. Assume that $\epsilon^{\prime} \in \mathbb{Z}$ and $N \in \mathbb{Z}^{+}$are fixed. Suppose $\epsilon \in \mathbb{Q}$ has the property that $N \epsilon \in \mathbb{Z}$. Then, for $\left(\begin{array}{l}a b \\ c d\end{array}\right) \in \mathrm{SL}(2, \mathbb{Z})$ with $\left(\begin{array}{ll}a b \\ c d\end{array}\right) \equiv\left(\begin{array}{ll}1 & 0 \\ 0 & 1\end{array}\right)(\bmod 2 N)$, we have

$$
\theta\left[\begin{array}{c}
\epsilon \\
\epsilon^{\prime}
\end{array}\right]\left(N\left(\frac{a \tau+b}{c \tau+d}\right)\right)=\lambda \sqrt{c \tau-d} \nu(\epsilon) \theta\left[\begin{array}{c}
\epsilon \\
\epsilon^{\prime}
\end{array}\right](N \tau),
$$

where $\nu(\epsilon)=e\left(\frac{-\epsilon^{2} N b(a-2)+2 \epsilon \epsilon^{\prime}(d-1)}{8}\right)$. If $\epsilon^{\prime}$ is odd, and $N$ and $N \epsilon$ have the same parity, then (A.1) still holds for $\left(\begin{array}{c}a b \\ c d\end{array}\right) \equiv\left(\begin{array}{ll}1 & * \\ 0 & 1\end{array}\right)(\bmod N)$, provided we take

$$
\nu(\epsilon)= \begin{cases}e\left(\frac{-\epsilon^{2} N b(a-2)}{8}\right), & \text { if } N \text { and } N \epsilon \text { are both odd } \\ e\left(\frac{-\epsilon^{2} N b(a-2)+2 \epsilon \epsilon^{\prime}(b c+d-1)}{8}\right), & \text { if } N \text { and } N \epsilon \text { are both even } .\end{cases}
$$

If $\epsilon^{\prime}, N$ and $N \epsilon$ are all even, then (A.1) holds for $\left(\begin{array}{c}a b \\ c d\end{array}\right) \equiv\left(\begin{array}{c}1 \\ 0\end{array}\right)(\bmod 2 N)$, provided we take $\nu(\epsilon)=e\left(\frac{-\epsilon^{2} N b(a-2)}{8}\right)$.

Proof. It follows from (4.5) that for any $\left(\begin{array}{c}a b \\ c d\end{array}\right) \in \mathrm{SL}(2, \mathbb{Z})$ with $c \equiv 0(\bmod N)$,

$$
\begin{gathered}
\theta\left[\begin{array}{c}
\epsilon \\
\epsilon^{\prime}
\end{array}\right]\left(N\left(\frac{a \tau+b}{c \tau+d}\right)\right)=\theta\left[\begin{array}{c}
\epsilon \\
\epsilon^{\prime}
\end{array}\right]\left(\frac{a(N \tau)+N b}{(c / N)(N \tau)+d}\right) \\
=\lambda \sqrt{c \tau-d} e\left(\frac{-\epsilon\left(N a b d+b c \epsilon^{\prime}\right)}{4}\right) e\left(\frac{-\epsilon^{2} N a b}{8}\right) \theta\left[\begin{array}{c}
a \epsilon+(c / N) \epsilon^{\prime}-a c / N \\
N b \epsilon+d \epsilon^{\prime}+N b d
\end{array}\right](N \tau) .
\end{gathered}
$$

Suppose that $\left(\begin{array}{l}a b \\ c d\end{array}\right) \equiv\left(\begin{array}{ll}1 & 0 \\ 0 & 1\end{array}\right)(\bmod 2 N)$. Clearly

$$
\begin{aligned}
a \epsilon+(c / N) \epsilon^{\prime}-a(c / N) & =\epsilon+2 \ell \\
\text { and } \quad N b \epsilon+d \epsilon^{\prime}+N b d & =\epsilon^{\prime}+2 m
\end{aligned}
$$


for some integers $\ell$ and $m$. Thus by (4.3) we get

$$
\begin{gathered}
\theta\left[\begin{array}{c}
\epsilon \\
\epsilon^{\prime}
\end{array}\right]\left(N\left(\frac{a \tau+b}{c \tau+d}\right)\right) \\
=\lambda \sqrt{c \tau-d} e\left(-\epsilon \frac{N b d(a-1)-\epsilon^{\prime}(b c+d-1)}{4}\right) e\left(\frac{-\epsilon^{2} N b(a-2)}{8}\right) \theta\left[\begin{array}{c}
{ }^{\prime} \\
\epsilon^{\prime}
\end{array}\right](N \tau) \\
=\lambda \sqrt{c \tau-d} e\left(\frac{-\epsilon^{2} N b(a-2)+2 \epsilon \epsilon^{\prime}(d-1)}{8}\right) \theta\left[\begin{array}{c}
\epsilon \\
\epsilon^{\prime}
\end{array}\right](N \tau) .
\end{gathered}
$$

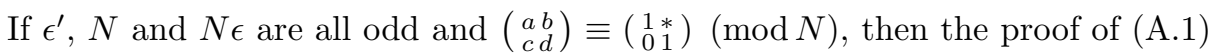
is similar but a little trickier. In (A.4) we write $a=1+N r$ and $c=N s$ for $r, s \in \mathbb{Z}$ and observe that if $r$ is odd, then $s$ must be odd, since $(a, c)=1$. Thus

$$
a \epsilon+(c / N) \epsilon^{\prime}-a(c / N)=\epsilon+r(N \epsilon-N s)+s\left(\epsilon^{\prime}-1\right) \equiv \epsilon \quad(\bmod 2),
$$

since if $r$ is odd, $N \epsilon-N s$ must be even. In A.5 we write $d=1+N t$ for $t \in \mathbb{Z}$ to get

$$
N b \epsilon+d \epsilon^{\prime}+N b d=\epsilon^{\prime}+b\left(N \epsilon+N^{2} t+N\right)+N t \epsilon^{\prime} \equiv \epsilon^{\prime}(\bmod 2),
$$

which is clear if $t$ is even, while if $t$ is odd, then $d$ is even and so $b$ is odd.

Proceeding as before we get (A.6), but now we observe that

$$
\operatorname{Nbd}(a-1)-\epsilon^{\prime}(b c+d-1)
$$

is always divisible by $2 N$. For this it is enough to show that it is even. But $a d-b c=1$ implies that there are among $a, b, c, d$ either two even numbers, which are either $a$ and $d$ or $b$ and $c$, or one even, and the rest odd. Checking all six cases gives the result. Thus we have that

$$
-N \epsilon \frac{N b d(a-1)-\epsilon^{\prime}(b c+d-1)}{2 N}
$$

is an integer whose parity does not depend on $\epsilon$, since $N \epsilon$ is odd. Returning to (A.6) we see that the first case of (A.2) holds.

The rest of the proof is similar but easier.

\section{REFERENCES}

[An1] G. E. Andrews, An introduction to Ramanujan's "lost" notebook. Amer. Math. Monthly 86 (1979), 89-108. MF0520571 (80e:01018)

[An2] G. E. Andrews, Ramanujan's "lost" notebook. III. The Rogers-Ramanujan continued fraction. Adv. in Math. 41 (1981), no. 2, 186-208. MR0625893 (83m:10034c)

[Art] E. Artin, Galois theory. Edited and with a supplemental chapter by Arthur N. Milgram. Reprint of the 1944 second edition, Dover, Mineola, NY, 1998. MR.1616156 (98k:12001)

[Ask] R. Askey, Orthogonal polynomials and theta functions. Theta functions-Bowdoin 1987, Part 2 (Brunswick, ME, 1987), 299-321, Proc. Sympos. Pure Math., 49, Part 2, Amer. Math. Soc., Providence, RI, 1989. MR1013179 (90h:33006)

[BC] S. Barnard and J.M. Child, Advanced Algebra. Macmillan, London, 1939. MR0001185 $(1: 193 a)$

[Be] B. C. Berndt, Ramanujan's notebooks. Parts I-V. Springer-Verlag, New York, 19851998. MR0781125 (86c:01062). MR1486573 (99f:11024)

[BCZ] B. C. Berndt, H. H. Chan and L-C. Zhang, Explicit evaluations of the Rogers-Ramanujan continued fraction. J. für die reine und angew. Math. 480 (1996), 141-159. MR1420561 (98c:11007)

[Ber] W. E. H. Berwick, Modular invariants expressible in terms of quadratic and cubic irrationalities. Proc. London Math. Soc. (2) 28, 53-69 (1928).

[Bir] B. J. Birch, Weber's class invariants, Mathematika 16, 283-294 (1969). MR0262206 $(41: 6816)$ 
[Bor] R. E. Borcherds, What is Moonshine? Proceedings of the International Congress of Mathematicians, Vol. I (Berlin, 1998). Doc. Math. 1998, Extra Vol. I, 607-615. MR.1660657 (99j:17001)

[BCHIS] A. Borel, S. Chowla, C. S. Herz, K. Iwasawa and J-P. Serre, Seminar on complex multiplication. Seminar held at the Institute for Advanced Study, Princeton, N.J., 195758. Lecture Notes in Mathematics, No. 21, Springer-Verlag, Berlin-New York, 1966. MR0201394 (34:1278)

[Bra] R. Brauer, Galois Theory, 1957-1958, Harvard Lecture Notes.

[Cox] D. A. Cox, Primes of the form $x^{2}+n y^{2}$. Fermat, class field theory and complex multiplication. John Wiley and Sons, Inc., New York, 1989. MR.1028322 (90m:11016)

[Dar] H. B. C. Darling, Proofs of certain identities and congruences enunciated by S. Ramanujan. Proc. Lond. M. S. (2) 19, 350-372 (1921).

[Deu] M. Deuring, Die Klassenkörper der komplexen Multiplikation, Enz. Math. Wiss., Band I-2, Heft 10, Teil II. B. G. Teubner Verlagsgesellschaft, Stuttgart, 1958. MR0167481 $(29: 4754)$

[Dic] L. E. Dickson, Modern algebraic theories. Sanborn, New York, 1926.

[Eis] G. Eisenstein, Theorema. J. für die reine und angew. Math. 29, 96-97 (1845) [ \#26 in Mathematische Werke. Band I. 289-290, Chelsea Publishing Co., New York, 1975].

[Elk] N. D. Elkies, The Klein quartic in number theory. The eightfold way, 51-101, Math. Sci. Res. Inst. Publ., 35, Cambridge Univ. Press, Cambridge, 1999. MR1722413 (2001a:11103)

[FK] H. Farkas and I. Kra, Theta constants, Riemann surfaces and the modular group. American Mathematical Society, Providence, RI, 2001. MR1850752 (2003a:11045)

[Fol] A. Folsom, Modular forms and Eisenstein's continued fractions. Preprint, 2004.

[Fri] R. Fricke, Lehrbuch der Algebra, Band 2, Vieweg, Braunschweig, 1926.

[Göl] H. Göllnitz, Partitionen mit Differenzenbedingungen. J. für die reine und angew. Math. 225 (1967), 154-190. MR0211973 (35:2848)

[Gor] B. Gordon, Some continued fractions of the Rogers-Ramanujan type. Duke Math. J. 32 (1965), 741-748. MR0184001 (32:1477)

[Har] G. H. Hardy, Ramanujan: twelve lectures on subjects suggested by his life and work. Chelsea Publishing Company, New York, 1959. MR0106147 (21:4881)

[Hei] E. Heine, Verwandlung von Reihen in Kettenbrüche. J. für die reine und angew. Math. 32, 205-209 (1846).

[Hir] M. D. Hirschhorn, On the expansion of Ramanujan's continued fraction. Ramanujan J. 2 (1998), no. 4, 521-527. MR1665326 (99i:11006)

[Jac] C. G. J. Jacobi, Allgemeine Theorie der kettenbruchaehnlichen Algorithmen, in welchen jede Zahl aus drei vorhergehenden gebildet wird, J. für die reine und angew. Math. 69, 29-64 (1869) [in Mathematische Werke, VI, pp. 385-426, Chelsea, New York, 1969].

[K11] F. Klein, Weitere Untersuchungen über das Ikosaeder. Math. Ann. 12, 509-561 (1877) [in Gesammelte Math. Abhandlungen II , 321-384, Springer, 1922].

[K12] F. Klein, Über die Transformation der elliptischen Functionen und die Auflösung der Gleichungen fünften Grades. Math. Ann. 14, 111-172 (1878) [in Gesammelte Math. Abhandlungen III, 13-75, Springer, 1922].

[K13] F. Klein, Über die Transformation siebenter Ordnung der elliptischen Functionen, Math. Ann. 14, 428-471 (1879) [in Gesammelte Math. Abhandlungen III, 90-136, Springer, 1922].

[K14] F. Klein, Lectures on the icosahedron and the solution of equations of the fifth degree. Translated into English by George Gavin Morrice. Second and revised edition, Dover Publications, Inc., New York, NY, 1956. MR0080930 (18:329c)

[Kno] M. I. Knopp, Modular functions in analytic number theory. Markham Publishing Co., Chicago, 1970. MR0265287 (42:198)

[Mag] W. Magnus, Vignette of a cultural episode. Studies in numerical analysis. 7-13, Academic Press, London, 1974. MR0347521 (50:24)

[MM] H. McKean and V. Moll, Elliptic curves. Function theory, geometry, arithmetic. Cambridge University Press, Cambridge, 1997. MR1471703 (98g:14032)

[Mu1] T. Muir, New general formula for the transformation of infinite series into continued fractions. Transactions of the Royal Society of Edinburgh, 27 (1876), 467-471. 
[Mu2] T. Muir, On Eisenstein's continued fractions, Transactions of the Royal Society of Edinburgh, 28 (1876-1878), 135-144.

[New] M. Newman, Classification of normal subgroups of the modular group. Trans. Amer. Math. Soc. 126 (1967), 267-277. MR.0204375 (34:4217)

[Ogg] A. P. Ogg, Rational points of finite order on elliptic curves. Invent. Math. 12 (1971), 105-111. MR0291084 (45:178)

[Per] O. Perron, Die Lehre von den Kettenbrüchen. Chelsea, New York, 1950. MR0037384 $(12: 254 \mathrm{~b})$

[Ra1] K. G. Ramanathan, On Ramanujan's continued fraction. Acta Arith. 43 (1984), no. 3, 209-226. MR0738134 (85d:11012)

[Ra2] K. G. Ramanathan, On some theorems stated by Ramanujan. Number theory and related topics (Bombay, 1988), 151-160, Tata Inst. Fund. Res. Stud. Math., 12, Tata Inst. Fund. Res., Bombay, 1989. MR 1441329 (98g:33027)

[Ram1] S. Ramanujan, Collected Papers, Chelsea, New York, 1962.

[Ram2] S. Ramanujan, Notebooks (2 volumes), Tata Institute of Fundamental Research, Bombay, 1957. MR0099904 (20:6340)

[Ram3] S. Ramanujan, The Lost Notebook and Other Unpublished Papers, Narosa, New Delhi, and Springer-Verlag, Berlin, 1988. MR0947735 (89j:01078)

[Ro1] L. J. Rogers, Second memoir on the expansion of certain infinite products, Proc. London Math. Soc. 25 (1894), 318-343.

[Ro2] L. J. Rogers, On a type of modular relation, Proc. London Math. Soc. (2) 19, 387-397 (1921).

[S] Th. Schneider, Arithmetische Untersuchungen elliptischer Integrale, Math. Ann. 113, 1-13 (1937)

[Sc] B. Schoeneberg, Elliptic modular functions: an introduction. Translated by J. R. Smart and E. A. Schwandt. Springer-Verlag, New York-Heidelberg, 1974. MR0412107 (54:236)

[Sch] I. Schur, Ein Beitrag zur additiven Zahlentheorie und zur Theorie der Kettenbrüche, Berl. Ber., 302-321 (1917) [\#28 in Gesammelte Abhandlungen, Band II, 117-136, SpringerVerlag, 1973].

[Se1] A. Selberg, Über einige arithmetische Identitäten. Avh. Norske Vidensk.- Akad. Oslo I, 1936, Nr. 8, 23 S. (1936) [\#1 in Collected papers, Vol. I. With a foreword by K. Chandrasekharan. Springer-Verlag, Berlin, 1989].

[Se2] A. Selberg, Reflections around the Ramanujan centenary, Ramanujan: essays and surveys, 203-213, Hist. Math., 22, Amer. Math. Soc., Providence, RI, 2001 [in Collected papers, Vol. II, Springer-Verlag, Berlin, 1991]. MR1862753, MR1001304 (90g:01055)

[Ser1] J-P. Serre, Extensions icosaédriques. Séminaire de Théorie des Nombres des Bordeaux, 1979-1980, Exp. No. 19 [\#123 in Collected papers, Volume III, Springer-Verlag, New York, 1985]. MR0604216 (83d:12003)

[Ser2] J-P. Serre, L'invariant de Witt de la forme $\operatorname{Tr}\left(x^{2}\right)$. Comment. Math. Helv. 59 (1984), no. 4, 651-676 [\#131 in Collected papers, Volume III, Springer-Verlag, New York, 1985]. MR0780081 (86k:11067)

[Ser3] J-P. Serre, Cohomological invariants, Witt invariants, and trace forms (notes by Skip Garibaldi). Univ. Lecture Ser., 28, Cohomological invariants in Galois cohomology, 1100, Amer. Math. Soc., Providence, RI, 2003. MR.1999384

[Shi] G. Shimura, Introduction to the arithmetic theory of automorphic functions. Princeton University Press, Princeton, NJ, 1994. MR.1291394 (95e:11048)

[Sil] J. H. Silverman, Advanced topics in the arithmetic of elliptic curves. Graduate Texts in Mathematics, 151, Springer-Verlag, New York, 1994. MR.1312368 (96b:11074)

[Sta] H. M. Stark, On complex quadratic fields wth class-number two. Collection of articles dedicated to Derrick Henry Lehmer on the occasion of his seventieth birthday. Math. Comp. 29 (1975), 289-302. MR0369313 (51:5548)

[Syl] J. J. Sylvester, On a remarkable modification of Sturm's theorem. Philosophical Magazine 4, 446-457 (1853) [\#61 in Collected Math. Papers, Vol. I, 609-619, Chelsea, New York, 1973].

[Tot] G. Toth, Finite Möbius groups, minimal immersions of spheres, and moduli. Universitext, Springer-Verlag, New York, 2002. MR,1863996 (2002i:53082)

[Wa1] G. N. Watson, Theorems stated by Ramanujan (VII): Theorems on continued fractions, J. London Math. Soc. 4 (1929), 39-48. 
[Wa2] G. N. Watson, Theorems stated by Ramanujan (IX): Two continued fractions, J. London Math. Soc. 4 (1929), 231-237.

[Web] H. Weber, Lehrbuch der Algebra, III. Braunschweig, 1908 [reprinted by Chelsea, New York, 1961].

Department of Mathematics, University of California, Box 951555, Los Angeles, CALifornia 90095-1555

E-mail address: wdduke@ucla.edu 